\title{
FIRST RESULTS FROM THE CATALINA REAL-TIME TRANSIENT SURVEY
}

\author{
A. J. Drake ${ }^{1}$, S. G. Djorgovski ${ }^{1}$, A. Mahabal ${ }^{1}$, E. Beshore ${ }^{2}$, S. Larson ${ }^{2}$, M. J. Graham ${ }^{1}$, R. Williams $^{1}$, E. Christensen ${ }^{3}$, \\ M. Catelan ${ }^{4,5,6}$, A. Boattini ${ }^{2}$, A. GibBs ${ }^{2}$, R. Hill $^{2}$, And R. Kowalski ${ }^{2}$ \\ ${ }^{1}$ California Institute of Technology, 1200 E. California Blvd., CA 91225, USA \\ ${ }^{2}$ Department of Planetary Sciences, The University of Arizona, Lunar and Planetary Laboratory, 1629 E. University Blvd., Tucson, AZ 85721, USA \\ ${ }^{3}$ Gemini Observatory, Casilla 603, La Serena, CL, Chile \\ ${ }^{4}$ Pontificia Universidad Católica de Chile, Departamento de Astronomía y Astrofísica, Av. Vicuña Mackena 4860, 782-0436 Macul, Santiago, Chile \\ ${ }^{5}$ Catholic University of America, Department of Physics, 200 Hannan Hall,Washington, DC 20064, USA \\ Received 2008 September 3; accepted 2009 February 12; published 2009 April 20
}

\begin{abstract}
We report on the results from the first six months of the Catalina Real-Time Transient Survey (CRTS). In order to search for optical transients (OTs) with timescales of minutes to years, the CRTS analyses data from the Catalina Sky Survey which repeatedly covers 26,000 of square degrees on the sky. The CRTS provides a public stream of transients that are bright enough to be followed up using small telescopes. Since the beginning of the survey, all CRTS transients have been made available to astronomers around the world in real time using HTML tables, RSS feeds, and VOEvents. As part of our public outreach program, the detections are now also available in Keyhole Markup Language through Google Sky. The initial discoveries include over 350 unique OTs rising more than 2 mag from past measurements. Sixty two of these are classified as supernovae ( $\mathrm{SNe}$ ), based on light curves, prior deep imaging and spectroscopic data. Seventy seven are due to cataclysmic variables (CVs; only 13 previously known), while an additional 100 transients were too infrequently sampled to distinguish between faint CVs and SNe. The remaining OTs include active galactic nucleus, blazars, high-proper-motions stars, highly variable stars (such as UV Ceti stars), and transients of an unknown nature. Our results suggest that there is a large population of $\mathrm{SNe}$ missed by many current SN surveys because of selection biases. These objects appear to be associated with faint host galaxies. We also discuss the unexpected discovery of white dwarf binary systems through dramatic eclipses.
\end{abstract}

Key words: BL Lacertae objects: general - galaxies: general - novae, cataclysmic variables - stars: flare supernovae: general

Online-only material: color figures

\section{INTRODUCTION}

Time-domain astronomy is one of the most rapidly emerging areas of astronomy (Paczyński 2000). Several large experiments such as the Large Synoptical Survey Telescope (LSST; Ivezic et al. 2008), the Panoramic Survey Telescope and Rapid Response System (PanSTARRS; Hodapp et al. 2004), and SkyMapper (Keller et al. 2007), are set to make a major impact in the near future by covering thousands of square degrees with targets ranging from distant (supernovae) $\mathrm{SNe}$ to nearEarth asteroids. Past time-domain surveys have mainly concentrated on areas of tens to hundreds of square degrees and have typically searched for specific types of astronomical transients such as microlensing (MACHO; Alcock et al. 2000; OGLE, Udalski et al. 1994; EROS, Aubourg et al. 1995), gamma-ray burst (GRB) afterglows (ROTSE; Akerlof et al. 2000), and SNe (Lick Observatory Supernova Search (LOSS); Filippenko et al. 2001).

Deep surveys for variability have been carried out over small areas $\left(<25 \mathrm{deg}^{2}\right)$ by the Subaru/XMM-Newton Deep Survey (SXDS; Morokuma et al. 2008), the Deep Lensing Survey (DLS; Becker et al. 2004), and the Faint Sky Variability Survey (FSVS; Huber et al. 2006) whereas large-area surveys, such as the Sloan Digital Sky Survey (SDSS; York et al. 2002), the Two Micron All Sky Survey (2MASS; Skrutskie et al. 2006), and the Galaxy Evolution Explorer (GALEX; Martin et al. 2005) have generally not been synoptic. One survey intermediate between past deep surveys and wide future surveys was carried out by the SDSS

\footnotetext{
6 John Simon Guggenheim Memorial Foundation Fellow.
}

consortium to discover Type Ia SNe. This survey covered 300 $\mathrm{deg}^{2}$ in five optical bands to $r \sim 23$ during nine months spaced over 2005-2007 (Sesar et al. 2007).

\subsection{Current Transient Surveys}

Current wide-field transient surveys include the Robotic Optical Transient Search Experiment (ROTSE-III; Akerlof et al. 2003) and the All Sky Automated Survey (ASAS-3; Pojmanski 2001). However, like many other current surveys, ROTSE-III is targeted toward the discovery and characterization of GRB afterglows. The ASAS-3 survey can cover the visible southern sky every two nights and covers $\sim 30,000 \mathrm{deg}^{2}$ to $V \sim 13.5$ during the year (Pojmanski 2001), while the four telescopes of the ROTSE-III survey routinely cover $1260 \mathrm{deg}^{2}$ to $R \sim 17.5$ (Rykoff et al. 2005; Yost et al. 2007).

In the time between targeted surveys, and future wide, deep, transient surveys, two experiments have been searching tens of thousands of square degrees of the sky for transients; the Palomar-Quest (PQ) ${ }^{7}$ digital synoptic sky survey (Djorgovski et al. 2008a), and the Catalina Real-Time Transient Survey (CRTS). The PQ survey started analyzing drift scan data for optical transients (OTs) in real time in August 2006, while the CRTS began in 2007 November. The transients discovered in PQ data will be presented in A. Mahabal et al. (2009a, in preparation).

Both CRTS and PQ experiments use a purpose-built pipeline for real-time transient detection, analysis and distribution (A. J.

\footnotetext{
7 http://palquest.org
} 
Drake et al. 2009, in preparation). These surveys are now set to provide estimates of the rates and types of OTs that can be expected from future synoptic surveys.

In these experiments, unlike most current and past OT surveys, the results are made public within minutes of discovery using the International Virtual Observatory Alliance (IVOA) standard VOEvent protocol and the VOEventNet astronomical event distribution network (Drake et al. 2007a). In addition, VOEventNet, in collaboration with Google, makes transient astronomy available to the world in real time via a dedicated layer in Google Sky. ${ }^{8}$ Current events available through VOEventNet also include MOA and OGLE microlensing, GCN GRB alerts, and PQ transients.

The CRTS routinely searches for OTs in data from the Catalina Sky Survey (CSS) Schmidt Telescope. In this paper, we present results from the first six months. First, we will discuss the observations and data analysis (Section 2). We will then list the current results (Section 3) and discuss some of the types of transients discovered. Finally, we will summarize the current findings (Section 4).

\section{OBSERVATIONS AND DATA REDUCTION}

The $\operatorname{CSS}^{9}$ (Larson et al. 2003) uses the $0.7 \mathrm{~m} f / 1.9$ Catalina Schmidt Telescope north of Tucson, Arizona to discover nearEarth objects (NEOs) and potentially hazardous asteroids (PHAs). This survey uses a single unfiltered $4 \mathrm{k} \times 4 \mathrm{k}$ CCD with 2".5 pixels, giving an $8 \mathrm{deg}^{2}$ field of view. To date, CSS has found hundreds of PHAs, comets and other solar system objects, and currently leads the rate of NEA discoveries. ${ }^{10}$ On a clear night, the Catalina Schmidt typically covers $1200 \mathrm{deg}^{2}$ of sky in a sequence of four $30 \mathrm{~s}$ exposures. For each observation, there is an additional $18 \mathrm{~s}$ overhead for readout, step and settle time.

Currently observed fields cover $\sim 26,000 \mathrm{deg}^{2}$ in the declination range $-30^{\circ}<\delta<70^{\circ}$. Observations are generally limited to Galactic latitudes $|b|>10^{\circ}$ to prevent confusion caused by crowding. Weather permitting, observations are made on 21-24 nights per lunation and typically reach $V$ magnitudes from 19 to 20. Observations are made in a grid of adjacent fields so that sets of four observations of a field are taken at equal intervals over $\sim 30$ minutes. This allows us to detect transients varying on timescales from minutes to years. In addition, the four image sequence provides a significant veto for asteroids and artifacts (due to cosmic rays and hot pixels) that often cannot be distinguished from genuine rapid transients in pairs of exposures.

In the near future, we hope to include two additional dedicated CSS telescopes in OT searches. The $1.5 \mathrm{~m} \mathrm{Mt}$. Lemmon reflector near Tucson, with a $1 \mathrm{deg}^{2}$ field of view, and the $0.5 \mathrm{~m}$ Uppsala Schmidt at Siding Spring, Australia, with a $4.2 \mathrm{deg}^{2}$ field of view. All three telescopes operate in the same mode with similar cameras and reduction software.

\subsection{Transient Detection}

The main goal of the CRTS thus far has been to discover and characterize variable source populations that exhibit highamplitude variability on the timescales probed by CSS data. In this paper, we define OTs as objects that vary in brightness by greater than 2 mag between past catalogs derived from high signal-to-noise ratio $(\mathrm{S} / \mathrm{N})$ co-added images. The OT characterization process undertaken here includes understanding known

\footnotetext{
8 http://earth.google.com/sky

9 http://www.lpl.arizona.edu/css/

10 http://neo.jpl.nasa.gov/stats/
}

types of variable objects, as well as searching for new kinds of OTs. At the same time, the results of this survey have been designed to deliver a transient discovery stream that is available to the entire astronomical community as a testbed for VOEventNet technology.

The CSS uses standard SExtractor photometry software to produce object catalogs for each image as it is read out. To find transients, we compare new detections against deep, clean source catalogs, rather than simply comparing these to catalogs from earlier observations. This is necessary as image artifacts and differences in image depth lead to an overwhelming number of unmatched detections. The CSS detections are primarily matched with objects detected in past CSS co-added data. Each co-add consists of the median combination of at least 20 images. These images reach sources approximately 2 mag fainter than the deepest individual CSS images (mag $\sim 22$ ). Thus, very few objects are missed in the reference catalog because of image artifacts or changes in depth and transparency. However, some objects are missing in the catalogs because they are blended in the co-added images. For this reason, the OT candidates are also matched against objects in the higher resolution USNO-B, SDSS, and PQ surveys.

An alternate method to catalog searches for finding transients is image subtraction. The image subtraction technique involves matching new observations to a high signal-to-noise template image before subtracting one from the other (Tomaney \& Crotts 1996; Drake et al. 1999). The resulting difference image is then searched for transients. Although this increases the noise associated with detections, this is a very effective way of revealing transient objects in the presence of significant flux from blended constant sources. For this reason, subtraction has been particularly successful for dense stellar fields observed in SN searches such used by the Nearby Supernova Factory (SNfactory; Aldering et al. 2002). However, this technique does not work well at blue wavelengths in broad filters because of atmospheric differential refraction (Drake et al. 1999). The resulting difference images have bipolar residuals at the locations of the stars and often lead to false detections and missed transients. The image subtraction process is also prone to fail, or produce artifacts, when images are taken in bad observing conditions. As catalog-based searches simply match lists of sources, they do not introduce new artifacts into the images, making them more suitable for unsupervised real-time transient searches.

In the PQ survey, we found that the detection of OTs in a single scan was hampered by the presence of large numbers of previously undiscovered asteroids (A. Mahabal et al. 2009a, in preparation). By design, the longer temporal separation of CSS images means that almost all asteroids can be detected from their motion between images. We currently require that a transient is detected in at least two observations in addition to requiring positional coincidence between images. This aids the removal of most false detections due to image artifacts such as hot pixels and cosmic rays. The four image sequences used by CSS to discover NEOs also allow us to search for objects that vary on timescales of minutes.

The structure of the CRTS data processing pipeline largely follows the PQ pipeline (A. J. Drake et al. 2009, in preparation). In short, all the CSS catalogs are processed as the images are taken, on site. Candidate OTs are filtered to remove detections associated with artifacts (such as saturation spikes) and moving objects (such as asteroids and satellite trails). After filtering, the transient cutout images, light curves, and associated metadata 
are posted to VOEventNet where the OTs are posted on webpages and an RSS feeds. ${ }^{11}$ Additional image cutouts (from SDSS, PQ, and the DSS) are added to the information about each OT candidate by VOEventNet. As one of the goals of CRTS is to enable rapid automated follow-up of short-timescale transients by robotic telescopes, VOEvent alerts are sent from the observatory as soon as the data is processed (approximately 5 minutes after the fourth observation in a sequence is obtained).

After the filtering process $\sim 1$ source in 200,000 is detected as a significant OT. All of these candidates are sent as VOEvents. Currently $\sim 50 \%$ of these are associated with clearly genuine OTs while others are mainly detections of coincident image artifacts, such as saturation spikes. The handful of transient candidates are screened by eye within minutes to hours of discovery and a updated list of transients is posted. ${ }^{12} \mathrm{We}$ expect to remove these image artifacts employing artificial neural networks as we used in the PQ survey.

\section{RESULTS}

In the first six months of the CRTS survey, images were searched covering a total $\sim 450,000 \mathrm{deg}^{2}$ from $\sim 2000,8 \mathrm{deg}^{2}$ fields. Over 350 OTs passed selection by eye. These included dozens of newly discovered cataclysmic variables (CVs; Drake et al., 2007b, 2008a, 2008c, 2008e, 2008g; Djorgovski et al. 2008b, 2008d; Glikman et al. 2008), flaring events (Drake et al., 2008d; Djorgovski et al. 2007), nearby high-proper-motion stars, blazars (Mahabal et al. 2008a), asteroids, and comets. In addition, although CRTS has not focused on SNe, many OTs have been confirmed as such, including SN 2007sr (Drake et al. 2007b), a bright SN in the Antennae galaxies. Fainter SNe have also been discovered (SN 2008au, 2008av, 2008al, 2008ba, 2008bb, 2008bm, 2008cg, 2008ck, 2008dc, 2008dd, 2008de, 2008df, 2008dk (Drake et al. 2007a, 2007b, 2008b, 2008d, 2008e, 2008f, 2008g, 2008h; and Mahabal et al. 2008a). Nine known SNe were also independently rediscovered by the CRTS pipeline (SNe 2006tf, 2007nm, 2007no, 2007pu, 2007qv, 2008aq, 2008aw, 2008ax, 2008dk).

Many types of OTs are readily distinguished in a small number of images. For instance, the presence of motion between images is the certain sign a transient is a nearby object. However, there are a number of cases where classification based on a single set of images is difficult. For instance, dwarf novae (DNe) type CVs can produce outbursts as large as 8 mag, rising to an absolute $V$-band magnitude of 2 (Harrison et al. 2004). The presence of such large outbursts means that faint $\mathrm{CV}$ systems may be undetectable at quiescence. In a similar way, SN can rise more than 20 mag and range between $-20<M_{V}<-16$ at peak brightness. SNe are a common endpoint of stellar evolution and can occur in undetected dwarf galaxies many magnitudes fainter than the SN itself. Although the absolute magnitudes of $\mathrm{DNe}$ and $\mathrm{SNe}$ are markedly different, in sparsely sampled data, both $\mathrm{DNe}$ and $\mathrm{SNe}$ associated with faint sources can be difficult to distinguish at discovery. For this reason, transients associated with bright galaxies are more likely to be followed as $\mathrm{SNe}$ candidates. Clearly, this kind of selection bias means that many $\mathrm{SNe}$ are never followed, and this may also have led to errors in determinations of SN rates. For instance, core collapse SNe (II, $\mathrm{Ib} / \mathrm{c}$ ) and Type Ia SN are well known to occur at different rates depending on galaxy morphology (Mannucci et al. 2008). The

\footnotetext{
$11 \mathrm{http}: / /$ voeventnet.caltech.edu/feeds/Catalina.shtml

$12 \mathrm{http}: / /$ nesssi.cacr.caltech.edu/catalina/Allns.html
}

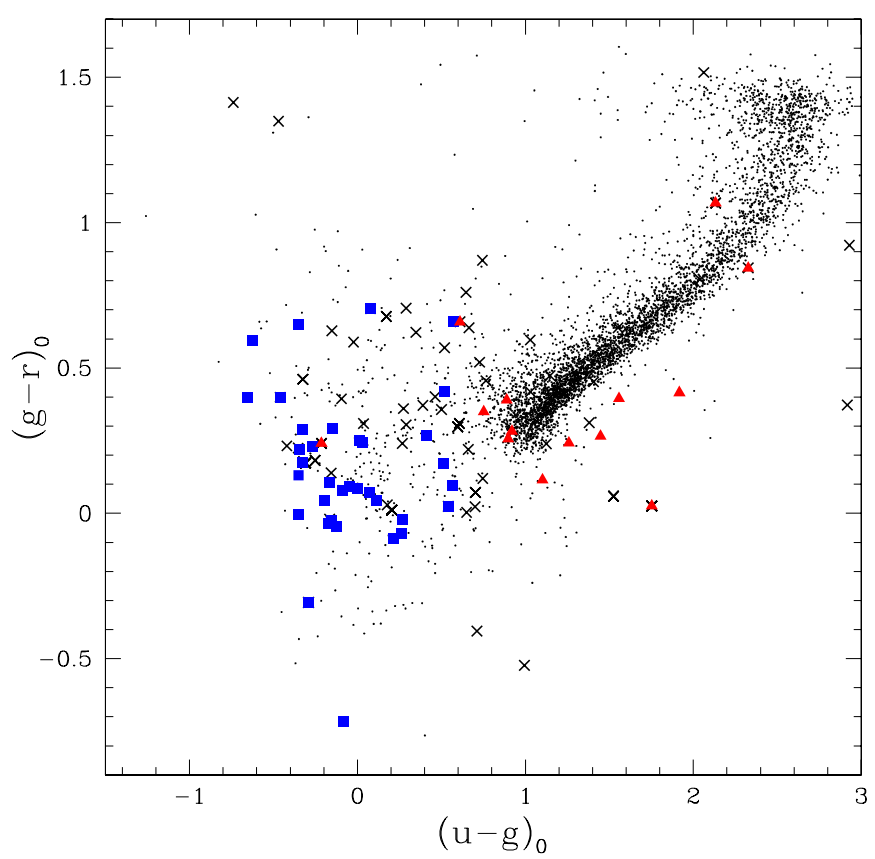

Figure 1. Color-color diagram of CRTS transients matching SDSS-DR6 sources. Triangles: likely and confirmed SN. Squares: confirmed and likely dwarf novae. Crosses: all other transients including possible SNe and DNe. The dots show the de-reddened stellar locus.

(A color version of this figure is available in the online journal.)

fact that few SNe are followed in faint galaxies may mean that the types of SNe occurring in these settings are underestimated.

$\mathrm{DNe}$ can readily be distinguished from $\mathrm{SNe}$ with spectroscopic observations. However, the number of telescopes capable of photometrically observing faint sources far outnumbers those which can spectroscopically monitor such sources. When spectroscopic observations are unavailable, other differences can be investigated. For instance, DNe outbursts typically last from few days to a couple of weeks, while $\mathrm{SNe}$ are bright for months. DNe outbursts take 1-3 days to rise to peak brightness while most SNe take 10-20 days. DNe recur on scales of days to years while $\mathrm{SNe}$ are one-time events. Both the DNe system and their outbursts are usually very blue while $\mathrm{SNe}$ pass through a range of colors as they age (depending on their type and host galaxy extinction). DNe can also be distinguished from SNe using accurate measurement of source color over time (Poznanski et al. 2002). Most CV systems can also be distinguished from variable stars on the giant branch and main-sequence stars in multiband photometry. For instance, from blue $(g-r)$ and $(r-i)$ colors in SDSS data (Kricinunas et al. 1998).

In Figure 1, we present the de-reddened color-color diagram of all the CRTS transients that match SDSS sources within 2.5. In this figure, we have separated $\mathrm{SNe}$ and $\mathrm{DNe}$ candidates with different symbols so that differences may be clearly seen. This figure shows that CVs are generally bluer than the $\mathrm{SNe}$ hosts in $(u-g)$ colours. The $(u-g)$ colours are consistent with those expected for white dwarfs (Eisenstein et al. 2006) and CVs discovered in SDSS data (Szkody et al. 2007). Since this figure only includes matches within 2.15 , it does not include the $\mathrm{SNe}$ discovered which were associated with large nearby galaxies. For these objects, the offsets from the galactic centers are much larger. The color separation of the two populations seen in Figure 1 tells us that if we see a source with $(u-g)<0.7$ it is much more likely to be due to a CV than an SN. Indeed of the 213 new and known CVs measured by Szkody et al. 


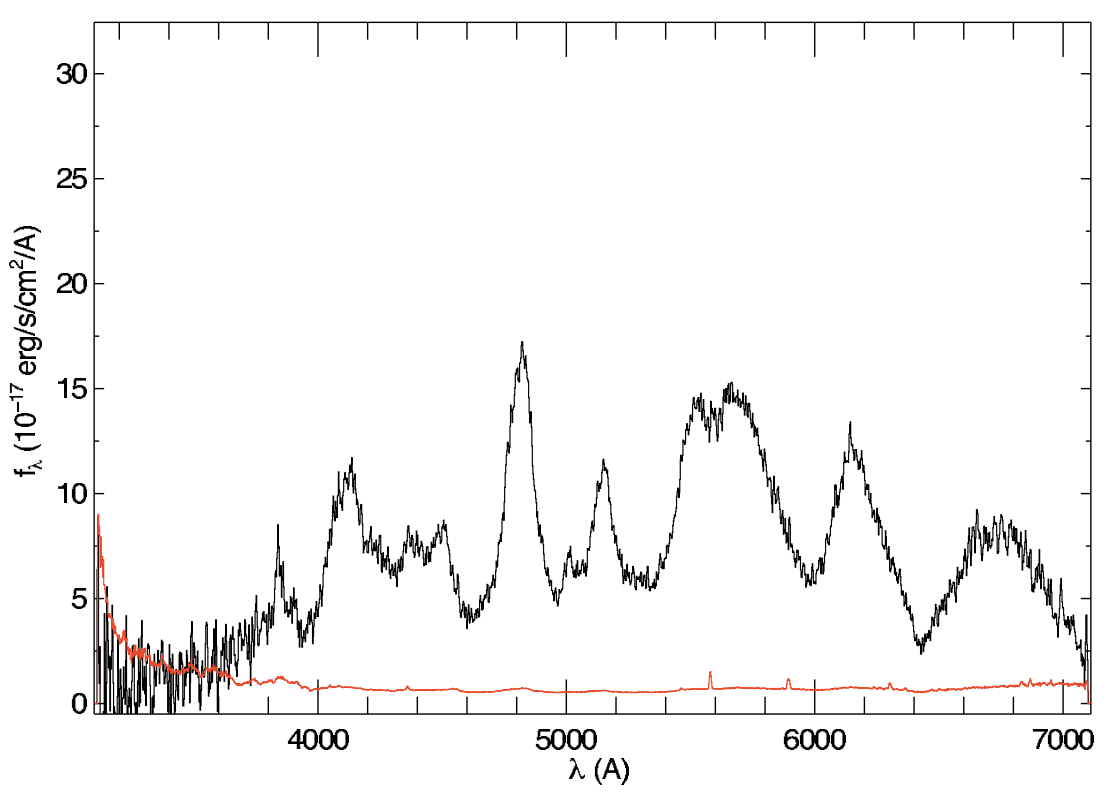

Figure 2. Palomar 200 inch DBMS spectrum of supernova SN $2008 \mathrm{ba}$.

(A color version of this figure is available in the online journal.)

$(2002,2003,2004,2005,2006,2007)$ in SDSS data, only 10 had $(u-g)>0.7$.

\subsection{Supernovae}

$\mathrm{SNe}$ are explosive endpoints of stellar evolution. In some cases, these objects can be used as cosmological standard candles that probe the depths of the expanding universe and aid our understanding dark energy. Many groups are currently searching for nearby SNe (LOSS; Filippenko et al. 2001, theSNfactory; Aldering et al. 2002, Chilean Automatic Supernova Search (CHASE); Pignata et al. 2007; Monard 2003; Puckett et al. 2003). Most of these groups only search for low- $z$ SNe clearly associated with large, bright, galaxies (Prieto et al. 2008) ${ }^{13}$. However, the SNfactory uses the wide-field images from the PQ survey to cover thousands of squares degrees (Wood-Vasey et al. 2004), like the CRTS.

In addition to the well known types of transients listed above, the CRTS discovered a large fraction of OTs that were either spatially associated with very faint blue galaxies, or had no visible source associated with them to the limits of PQ and SDSS surveys (greater than 3.5 mag fainter than the discovery images). One well known source of transients associated with galaxies is active galactic nucleus (AGN) flares (Totani et al. 2005). AGNs are known to contaminate SNe surveys (Gal-Yam et al. 2008; Sand et al. 2008) and vary on timescales from hours to years, with outbursts generally being up to $\sim 1 \mathrm{mag}$ (Webb $\&$ Malkan 2000). We expected to find a number of events from such objects. In contrast, the transients we discovered became many magnitudes brighter than their hosts over a period of 2 weeks and were visible for more than a month, consistent with $\mathrm{SNe}$. We carried out photometric follow-up of a number of these candidates and found that their colours and decline rates were indeed consistent with $\mathrm{SNe}$, rather than $\mathrm{AGN}, \mathrm{CV}$ s, or other variables stars (Poznanski et al. 2002). The most likely sources of such transients are $\mathrm{SNe}$ in very faint host galaxies or associated with intracluster stars.

\footnotetext{
13 http://www.supernovae.net
}

To select $\mathrm{SNe}$ from among CRTS transients, we removed asteroids near stationary points, flare stars, and DNe with faint sources. This was achieved by only selecting transients present at the same location on multiple nights spanning more that 2 weeks. In addition, we required that the objects were at least 2 mag brighter than any possible host, and that the transients faded slowly (as expected for $\mathrm{SNe}$ ). To investigate the accuracy of the classification based on six months of data, we reviewed archival CSS photometry going back more than three years and found no evidence of contamination by repeated outbursts of variable objects. The SNe candidates are given in Table 1.

Follow-up spectroscopy of two of the CRTS candidates associated with faint galaxies by Djorgovski et al. (2008c) revealed a Type Ia SN (SN 2008ba, $z \sim 0.03$ ) and a Type II SN (SN 2008bb, $z \sim 0.03$ ). In Figure 2, we present the spectrum of SN 2008ba. In Figure 3, we show the locations of the SNe in much deeper SDSS $r$-band images. SN 2008ba is $\sim 4^{\prime \prime}$ from an SDSS-identified galaxy with magnitude $m_{r}=21.5$. There are no other bright galaxies within $2^{\prime}$ of this object. As the redshift of this $\mathrm{SN}$ is 0.03 , the host galaxy must be fainter than $M_{r} \sim-13.9$. For SN 2008bb, there are also no bright $\left(m_{r}<19\right)$ galaxies within $2^{\prime}$ and no galaxies with $m_{R}<23$ within 0.5 (Figure 2, right). Assuming the brightest galaxy missed by SDSS to have $m_{r} \sim 23$, the host would have to be $M_{r}>-12.9$ at the SN's redshift (0.03).

In addition to the confirmed $\mathrm{SNe}$, we have discovered dozens of similar, long-timescale OTs. Seventeen of these have no sources present in CSS, PQ, or SDSS images overlapping the discovery locations (to magnitudes $\sim 21-23$ ). The remaining 24 match with faint galaxies that are $>2$ mag fainter than the transients associated with them. In Figure 4, we present an example of an SN where a faint host galaxy is detected, and in Figure 5, an SN where no host is seen to $r \sim 23$. The light curves corresponding to these OTs are given in Figure 6.

$\mathrm{SNe}$ with faint host galaxies are not unknown. For example, Prieto et al. (2008) studied recent nearby SN and found the SN Ic, $2007 \mathrm{bg}$, to be associated with a faint $\left(M_{B} \sim-12\right)$, very metalpoor $\left(\sim \frac{1}{20}\right.$ solar) host. Similarly, the PQ experiment discovered a Type Ic SN (2007nm) with an $m_{R} \sim 22$ host in DPOSS 
Table 1

Confirmed and Candidate Supernovae

\begin{tabular}{|c|c|c|c|c|c|c|c|}
\hline ID & $\begin{array}{c}\text { R.A. } \\
\text { (h:m:s) }\end{array}$ & $\begin{array}{c}\text { Dec } \\
\left(0^{\prime}, 1\right)\end{array}$ & $\begin{array}{l}\text { Date } \\
M J D\end{array}$ & Mag & $\operatorname{Mag}_{H}$ & Designation & Type \\
\hline CSS080514:145613+185115 & $14: 56: 13.27$ & $+18: 51: 15.4$ & 54600.18572 & 18.4 & 23 & $2008 \mathrm{dk}$ & $\mathrm{Ia}^{\mathrm{a}}$ \\
\hline CSS080508:112311+341251 & $11: 23: 10.90$ & $+34: 12: 51.0$ & 54594.27706 & 18.4 & 16.4 & $2008 \mathrm{cz}$ & Ia \\
\hline CSS080505:160625+100521 & 16:06:25.06 & $+10: 05: 21.4$ & 54591.37061 & 18.8 & 21.8 & $2008 \mathrm{df}$ & $\ldots$ \\
\hline CSS080505:155415+105825 & $15: 54: 15.15$ & $+10: 58: 25.0$ & 54591.37006 & 16.7 & 16.8 & $2008 \mathrm{cg}$ & Ia \\
\hline CSS080429:132846+251644 & $13: 28: 46.47$ & $+25: 16: 44.2$ & 54585.25061 & 18.6 & 21.2 & $\ldots$ & $\ldots$ \\
\hline CSS080427:152242+302208 & $15: 22: 42.30$ & $+30: 22: 08.0$ & 54583.39125 & 18.5 & 21.7 & $2008 \mathrm{ck}$ & Ia \\
\hline CSS080426:084328+302346 & $08: 43: 27.86$ & $+30: 23: 45.6$ & 54582.15121 & 18.2 & $>23$ & $\ldots$ & $\ldots$ \\
\hline CSS080417:155525-094147 & $15: 55: 24.97$ & $-09: 41: 47.1$ & 54573.37332 & 16.7 & $21^{\mathrm{c}}$ & 2008de & II \\
\hline CSS080415:122958+283538 & $12: 29: 57.71$ & $+28: 35: 38.1$ & 54571.16946 & 19.5 & $>23$ & $\ldots$ & $\ldots$ \\
\hline CSS080414:134142-162022 & $13: 41: 42.18$ & $-16: 20: 21.8$ & 54570.31002 & 18.4 & $21^{\mathrm{c}}$ & $\ldots$ & $\ldots$ \\
\hline CSS080409:170404+213542 & 17:04:03.60 & $+21: 35: 42.0$ & 54565.34357 & 17.7 & 20.1 & 2008dd & $\ldots$ \\
\hline CSS080406:164729+091826 & $16: 47: 29.45$ & $+09: 18: 25.8$ & 54562.46315 & 18.1 & $21^{\mathrm{c}}$ & $2008 \mathrm{dc}$ & $\mathrm{Ib} / \mathrm{c}$ \\
\hline CSS080406:122515+064526 & $12: 25: 15.03$ & $+06: 45: 26.2$ & 54562.35553 & 18.9 & $>23$ & $\ldots$ & $\ldots$ \\
\hline CSS080405:155205-000627 & $15: 52: 04.82$ & $-00: 06: 27.0$ & 54561.46586 & 18.7 & 21.2 & $\ldots$ & $\ldots$ \\
\hline CSS080404:130414-101913 & 13:04:14.11 & $-10: 19: 12.9$ & 54560.34851 & 15.1 & 11.8 & 2008aw & II \\
\hline CSS080404:125030-105201 & $12: 50: 30.36$ & $-10: 52: 01.3$ & 54560.34718 & 15.9 & 13.1 & 2008aq & $\mathrm{IIb}$ \\
\hline CSS080403:123041+413816 & $12: 30: 40.80$ & $+41: 38: 16.1$ & 54559.20966 & 13.3 & 9.4 & $2008 a x$ & IIn \\
\hline CSS080401:092227+282421 & 09:22:27.05 & $+28: 24: 20.8$ & 54557.22644 & 18.7 & $23^{*}$ & $\ldots$ & $\ldots$ \\
\hline CSS080330:121539+033613 & $12: 15: 39.10$ & $+03: 36: 12.9$ & 54555.30168 & 18.8 & 20.6 & $\ldots$ & $\ldots$ \\
\hline CSS080330:124441+051438 & $12: 44: 41.47$ & $+05: 14: 37.6$ & 54555.30276 & 19.2 & 21.4 & $\ldots$ & $\ldots$ \\
\hline CSS080329:130259+103027 & 13:02:58.75 & $+10: 30: 27.0$ & 54554.30700 & 17.2 & 15.8 & $2008 \mathrm{bm}$ & IIn \\
\hline CSS080313:132560-020027 & $13: 25: 59.69$ & $-02: 00: 27.1$ & 54538.36493 & 18.6 & 22.1 & $\ldots$ & $\ldots$ \\
\hline CSS080312:140609+242013 & 14:06:08.61 & $+24: 20: 12.5$ & 54537.39966 & 19.4 & $>23$ & $\ldots$ & $\ldots$ \\
\hline CSS080312:102245+021753 & $10: 22: 44.65$ & $+02: 17: 52.5$ & 54537.27925 & 18.9 & 21.7 & $\ldots$ & $\ldots$ \\
\hline CSS080310:112544+182317 & $11: 25: 44.03$ & $+18: 23: 17.1$ & 54535.36676 & 20.0 & $>23$ & $\ldots$ & $\ldots$ \\
\hline CSS080308:090908+111115 & 09:09:07.99 & $+11: 11: 15.3$ & 54533.20757 & 18.4 & 21.2 & $\ldots$ & $\ldots$ \\
\hline CSS080303:081413+271939 & $08: 14: 13.15$ & $+27: 19: 39.0$ & 54528.11129 & 18.1 & $23^{*}$ & $\ldots$ & $\ldots$ \\
\hline CSS080303:075520+203908 & $07: 55: 19.52$ & $+20: 39: 08.4$ & 54528.10484 & 19.3 & $>23$ & $2008 \mathrm{bb}$ & II \\
\hline CSS080302:124132+332203 & $12: 41: 31.98$ & $+33: 22: 03.2$ & 54527.29264 & 19.2 & $22.5^{*}$ & $\ldots$ & $\ldots$ \\
\hline CSS080302:145726+232348 & $14: 57: 26.21$ & $+23: 23: 48.2$ & 54527.47633 & 18.0 & $>22$ & 2008av & $\ldots$ \\
\hline CSS080228:142427-063345 & $14: 24: 26.63$ & $-06: 33: 44.8$ & 54524.48530 & 18.3 & $21^{*}$ & $\ldots$ & $\ldots$ \\
\hline CSS080228:105301-075656 & 10:53:00.81 & $-07: 56: 55.9$ & 54524.30812 & 19.2 & $>22$ & $\ldots$ & $\ldots$ \\
\hline CSS080227:160549+192717 & $16: 05: 49.17$ & $+19: 27: 17.2$ & 54523.47442 & 17.8 & 22 & 2008ba & Ia \\
\hline CSS080227:113034+130905 & $11: 30: 33.96$ & $+13: 09: 05.3$ & 54523.31151 & 18.7 & $>23$ & 2008au & $\ldots$ \\
\hline CSS080219: $151457+234110$ & $15: 14: 56.75$ & $+23: 41: 10.1$ & 54515.45977 & 16.6 & $>23$ & $\ldots$ & $\ldots$ \\
\hline CSS080218:134903+315237 & $13: 49: 03.35$ & $+31: 52: 37.1$ & 54514.41836 & 19.0 & 21.8 & $\ldots$ & $\ldots$ \\
\hline CSS080213:024607-073834 & 02:46:07.23 & $-07: 38: 33.8$ & 54509.09948 & 17.3 & 14.4 & 2008al & II \\
\hline CSS080210:080306+115121 & 08:03:06.09 & $+11: 51: 20.8$ & 54506.19556 & 18.8 & $>23$ & $\ldots$ & $\ldots$ \\
\hline CSS080206:130745+060805 & 13:07:44.64 & $+06: 08: 05.0$ & 54502.43974 & 19.8 & $>23$ & $\ldots$ & $\ldots$ \\
\hline CSS080111:070447+412518 & 07:04:47.34 & $+41: 25: 18.0$ & 54476.25764 & 19.0 & $>21$ & $\ldots$ & $\ldots$ \\
\hline CSS080111:145436-051254 & $14: 54: 35.97$ & $-05: 12: 53.8$ & 54476.49167 & 18.1 & $21^{*}$ & $\ldots$ & $\ldots$ \\
\hline CSS080101:035151-062700 & $03: 51: 50.64$ & $-06: 27: 00.5$ & 54466.25782 & 18.3 & $>23$ & $\cdots$ & $\cdots$ \\
\hline CSS080101:085249-052247 & $08: 52: 48.90$ & $-05: 22: 46.8$ & 54466.37420 & 19.1 & 20.5 & $\ldots$ & $\ldots$ \\
\hline CSS071230:082550+220041 & $08: 25: 50.48$ & $+22: 00: 41.3$ & 54464.35765 & 17.6 & 21.3 & $\ldots$ & $\ldots$ \\
\hline CSS071230:081807+200719 & 08:18:07.05 & $+20: 07: 19.2$ & 54464.32780 & 18.1 & 22 & $\cdots$ & $\ldots$ \\
\hline CSS071219:223508-011160 & $22: 35: 08.45$ & $-01: 11: 59.6$ & 54453.08583 & 18.3 & $>23$ & $2007 q v$ & II \\
\hline CSS071218:120153-185822 & $12: 01: 52.80$ & $-18: 58: 21.7$ & 54452.53386 & 12.9 & 9.8 & $2007 \mathrm{sr}$ & Ia \\
\hline CSS071216:121630+102303 & $12: 16: 29.63$ & $+10: 23: 02.9$ & 54450.44569 & 18.9 & 21.5 & $\ldots$ & $\ldots$ \\
\hline CSS071206:102130+200007 & $10: 21: 30.15$ & $+20: 00: 07.4$ & 54440.42300 & 18.6 & $21^{\mathrm{c}}$ & $\ldots$ & $\ldots$ \\
\hline CSS071204:035956+233018 & 03:59:56.34 & $+23: 30: 18.2$ & 54438.21532 & 18.5 & $>22$ & $\ldots$ & $\ldots$ \\
\hline CSS071204:002043+102044 & $00: 20: 42.71$ & $+10: 20: 44.1$ & 54438.13146 & 18.2 & $>22$ & $\ldots$ & $\ldots$ \\
\hline CSS071117:224558-003854 & $22: 45: 58.17$ & $-00: 38: 54.3$ & 54421.10263 & 18.6 & 20.7 & 2007pu & Ia \\
\hline CSS071112:012312+120818 & $01: 23: 12.27$ & $+12: 08: 18.0$ & 54416.17262 & 19.0 & $22^{\mathrm{c}}$ & $\ldots$ & $\ldots$ \\
\hline CSS071111:095436+045612 & 09:54:35.98 & $+04: 56: 11.8$ & 54415.46121 & 19.0 & 22.6 & $\ldots$ & $\cdots$ \\
\hline CSS071109:003334+214127 & $00: 33: 34.36$ & $+21: 41: 26.7$ & 54413.27788 & 18.8 & 16.1 & $\ldots$ & $\ldots$ \\
\hline CSS071107:093912+301341 & 09:39:11.62 & $+30: 13: 41.5$ & 54411.43569 & 18.2 & 20.0 & $\ldots$ & $\ldots$ \\
\hline CSS071103:224527+103933 & $22: 45: 27.41$ & $+10: 39: 32.7$ & 54407.18815 & 17.8 & $>21$ & $2007 \mathrm{~nm}$ & Ia \\
\hline CSS071102:024106-033814 & 02:41:05.71 & $-03: 38: 13.6$ & 54406.26445 & 19.6 & $>22$ & 2007no & Ia \\
\hline CSS071102:024457-044918 & $02: 44: 57.22$ & $-04: 49: 17.7$ & 54406.26445 & 18.8 & $>22$ & $\ldots$ & $\ldots$ \\
\hline CSS071101:015607+203404 & 01:56:06.60 & $+20: 34: 03.5$ & 54405.26126 & 18.8 & $>22$ & $\ldots$ & $\ldots$ \\
\hline CSS070320:124616+112555 & $12: 46: 15.81$ & $+11: 25: 55.4$ & 54179.37725 & 17.6 & 20.8 & $2006 \mathrm{tf}$ & $\mathrm{IIn}^{\mathrm{b}}$ \\
\hline
\end{tabular}

Notes. Column 1, CSS ID; Columns 2 and 3, right ascension and declination J2000; Column 4, modified Julian date of detection; Column 5, detection magnitude; Column 6, approximate source galaxy Gunn $r$ magnitude; Column 7, IAU designation.

a Sand et al. 2008, http://www.cfa.harvard.edu/iau/cbet/001400/CBET001410.txt. However, first announced and spectroscopically confirmed by the Supernova Factory as SNF20080510-001.

${ }^{\mathrm{b}}$ Found in archival data.

${ }^{\mathrm{c}}$ Estimated $r$-band magnitude.

images and redshift $z=0.04$. At this redshift, the host galaxy would have to be $M_{R}>-13.8$. Another possibility is that $\mathrm{SNe}$ without visible hosts could be associated with intracluster stars. Past searches for such SNe have been carried out by Sand et al. (2008) and Gal-Yam et al. (2008). As part of the WOOTS survey, Gal-Yam et al. $(2003,2008)$ discovered 12 SNe in Abell 

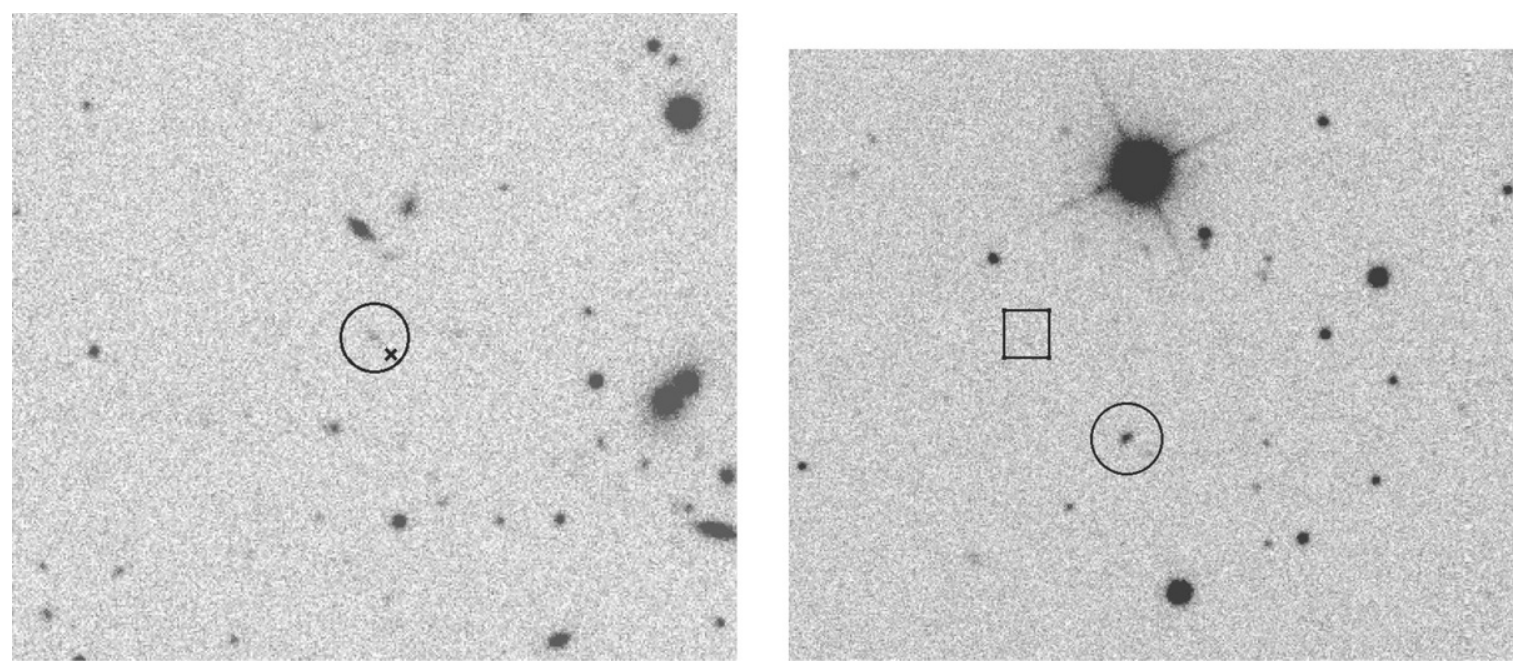

Figure 3. Left: SDSS prediscovery Gunn $r$-band image of the location of SN2008ba. The circle shows the location of the probable host, while the cross marks the location of the supernova. Right: SDSS prediscovery Gunn $r$-band image of the location of SN2008bb. The box shows the location of the SN, while the circle shows the location of the nearest SDSS galaxy.
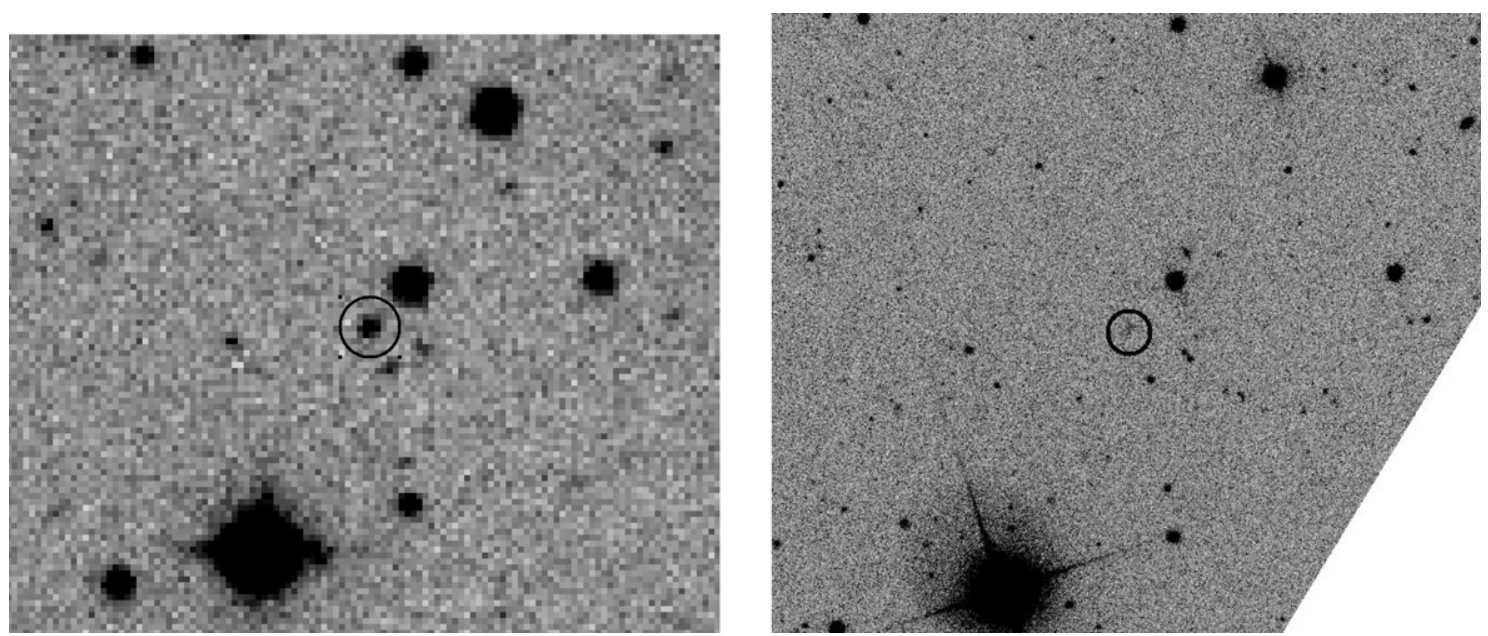

Figure 4. Left: 2007 December 30 CSS discovery image, showing the location of OT CSS071230:082550+220041 (circle). Right: SDSS Gunn $r$ image, showing the location of CSS071230:082550+220041 and the corresponding faint galaxy host.

clusters selected according to $0.06<z<0.2$. Two of these were identified as being intracluster SN Ia (SN $1998 \mathrm{fc}$ and SN 2001al). They found upper limits to possible dwarf hosts of $M_{r}>-14$ and -11.8 , respectively. Sand et al. (2008) discovered four photometrically selected intracluster SN with hosts $M_{g}>-14.3$. Based on this, they found $20 \%$ of stellar mass may reside in intracluster stars and thus $20 \%$ of the SN Ia parent stellar population could be intergalactic.

Interestingly, Stanek et al. (2006) and Savaglio et al. (2008) found that the long-duration GRBs associated with $\mathrm{SNe}$ are in faint, metal-poor, star-forming dwarf galaxies. This result was supported by Modjaz et al. (2008) who found that the galaxies containing Type Ic SNe that were not associated with GRBs, were significantly more metal rich than those with associated GRBs. Savaglio et al. (2008) also found that the host galaxies associated with GRBs are low-mass star-forming objects. The rate of $\mathrm{SNe}$ in such low-mass galaxies is expected to be low, as Kauffmann et al. (2003) found that only 13\% of the stellar mass in galaxies is found in objects less massive than $10^{9} M_{\odot}$. Such galaxies have only tens of millions of stars in contrast to large galaxies like our own with hundreds of billions of stars. For the hosts of SNe candidates not to be visible, most of these must be associated with low stellar-mass galaxies.

\subsubsection{Supernovae with Faint Galaxy Hosts?}

In order to test whether some of our $\mathrm{SNe}$ candidates are associated with galaxy clusters, we compared their locations to Abell clusters (Abell et al. 1989) and DPOSS clusters (GalYam et al. 2003). Two of the $48 \mathrm{SNe}$ in the DPOSS catalog region (with Galactic latitude $b>30^{\circ}$ ) matched the locations of DPOSS clusters and two matched Abell clusters. With only four matches to 12,000 clusters it appears very unlikely that the $\mathrm{SNe}$ are associated with such clusters. The SNfactory, like CRTS, uses wide-field images to find $\mathrm{SNe}$ rather than concentrating on bright galaxies like most other nearby $\mathrm{SNe}$ surveys. Therefore, both surveys have similar spatial sensitivity. We compared our results with 95 confirmed SNe from the Supernova Factory. ${ }^{14}$ Once again only two SNfactory SNe of the $63 \mathrm{SNe}$ with $b>30^{\circ}$ matched DPOSS clusters and two matched Abell clusters.

To determine whether the galaxies associated with our $\mathrm{SNe}$ candidates were indeed low-luminosity hosts, we calculated the difference between the peak measured magnitude in CRTS data and the host $R$-band magnitude. To provide upper limits to the host galaxies brightness where no host was visible,

$\overline{14}$ http://snfactory.lbl.gov/snf/open_access/login.php 

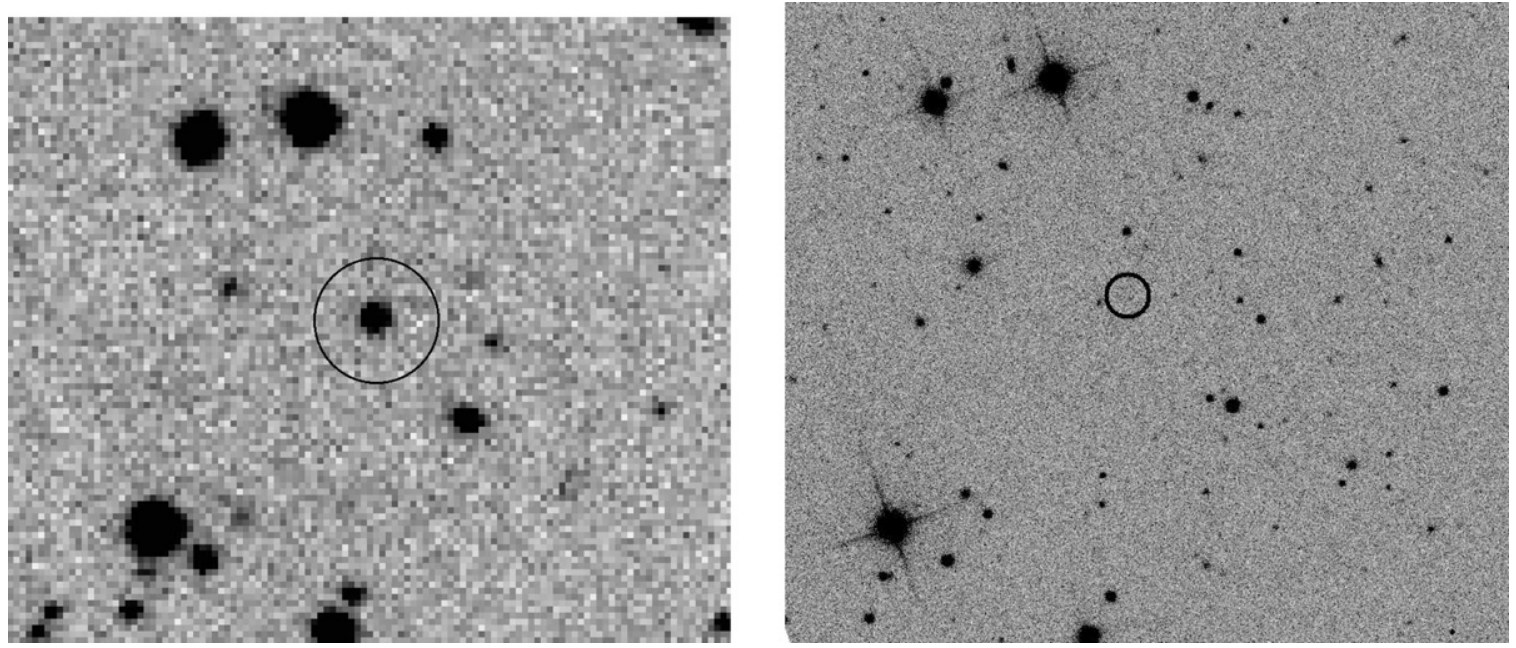

Figure 5. Left: 2008 January 1 CSS image, showing the location of OT CSS080219:151457+234110 (circle). Right: SDSS Gunn $r$ image of the same field. At the location of CSS080219:151457+234110, no host galaxy is visible.
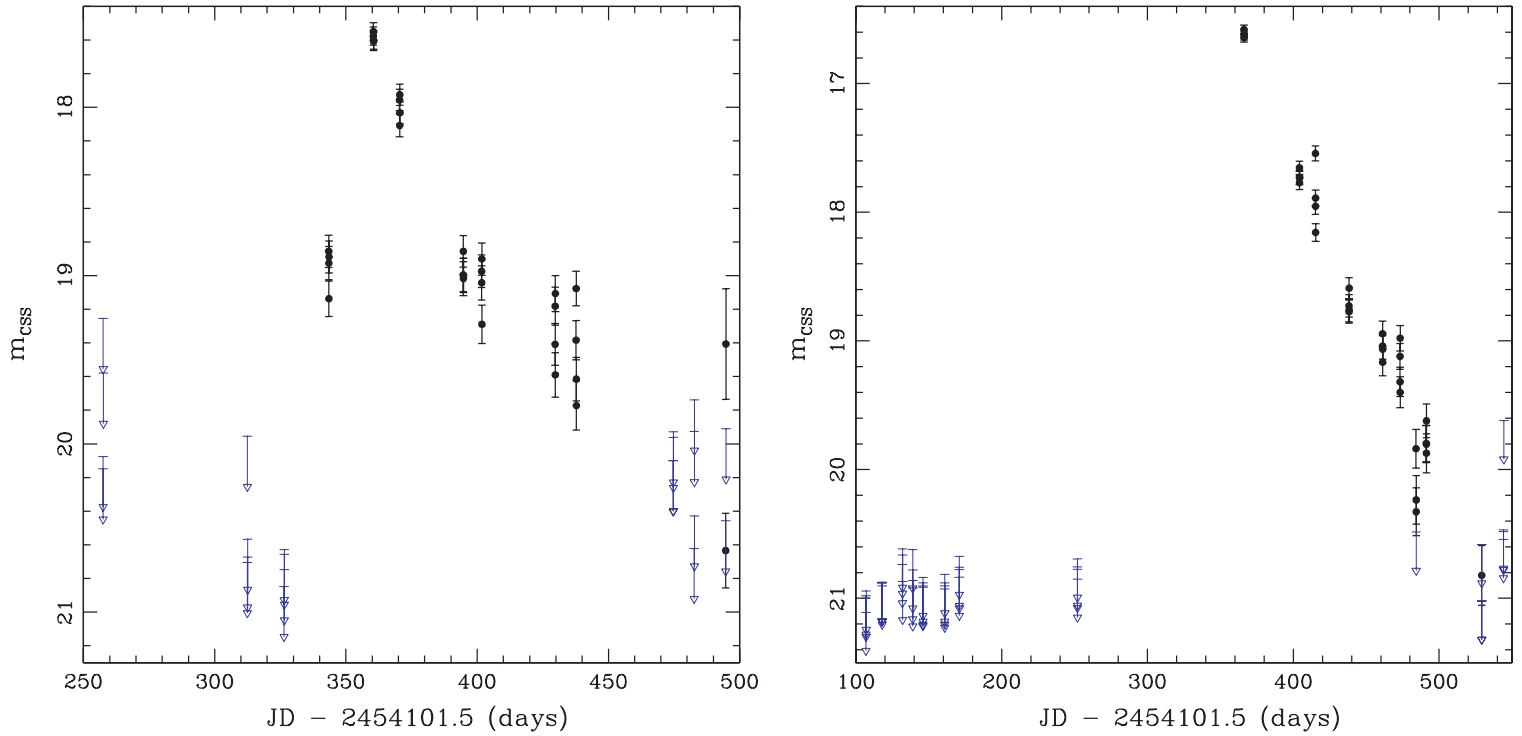

Figure 6. Left: the CRTS light curve of object CSS071230:082550+220041. Right: the CRTS light curve of object CSS080219:151457+234110. The black points are measurements and blue triangles nondetection upper limits.

(A color version of this figure is available in the online journal.)

the hosts were assumed to reside at the limits of the deepest observations covering them. Next, for the SNe with known spectral types (hence known peak absolute magnitude), we added the CRTS magnitude to the peak expected for the type of SNe. This provides a rough estimate of the host's absolute $R$-band magnitude. To test the SNe hosts where we do not have known spectral types we carried out the same process and assumed the $\mathrm{SNe}$ were all the brightest, common SN type, Ia. As the actual $\mathrm{SNe}$ types should be the same or fainter than our assumption, we obtain an approximate upper limit to the brightness of the associated host galaxies. For comparison, we took the SNfactory SNe and matched them with SDSS DR6 to find their host magnitudes. Fifty four lie within the region covered by SDSS DR6 and 52 match SDSS objects. Twenty nine of these hosts have spectra from which the redshift has been determined. For the other $23 \mathrm{SNe}$, we used the redshift of the SNe given by the SNfactory webpage. We then used the SDSS-DR6 $r$-band magnitudes and redshifts do derive the absolute magnitudes of the host galaxies assuming $H_{0}=72 \mathrm{~km} \mathrm{~s}^{-1} \mathrm{Mpc}^{-1}$.
In Figure 7, we plot histograms of the host galaxy magnitudes, for those with confirmed types and these combined with candidates (assumed Type Ia) as well as those with host from the SNfactory. This figure strongly suggests that the host galaxies must be intrinsically faint. The spectroscopically confirmed $\mathrm{SNe}$, that provide the most accurate host galaxy magnitudes, clearly reside in very faint hosts. The fact that the unconfirmed $\mathrm{SN}-$ host distribution peaks at a brighter magnitude is expected because of our assumption that these SNe were all bright Type Ia, rather than a mixture of types. Also, it is apparent that the SNfactory finds $\mathrm{SNe}$ in intrinsically brighter galaxies.

It is clear that by only selecting transients that are $2 \mathrm{mag}$ brighter than the sources associated with them, we will introduce a bias toward discovering $\mathrm{SNe}$ in faint galaxies, opposite to $\mathrm{SNe}$ surveys that have chosen only to follow bright galaxies. However, the CRTS SNe can also be discovered in large galaxies when they rise more than 2 mag above the local background. For intermediate magnitude hosts, the incremental increase in brightness is insufficient for $\mathrm{SNe}$ to be detected as separate sources. Detection of these would require the use of image 


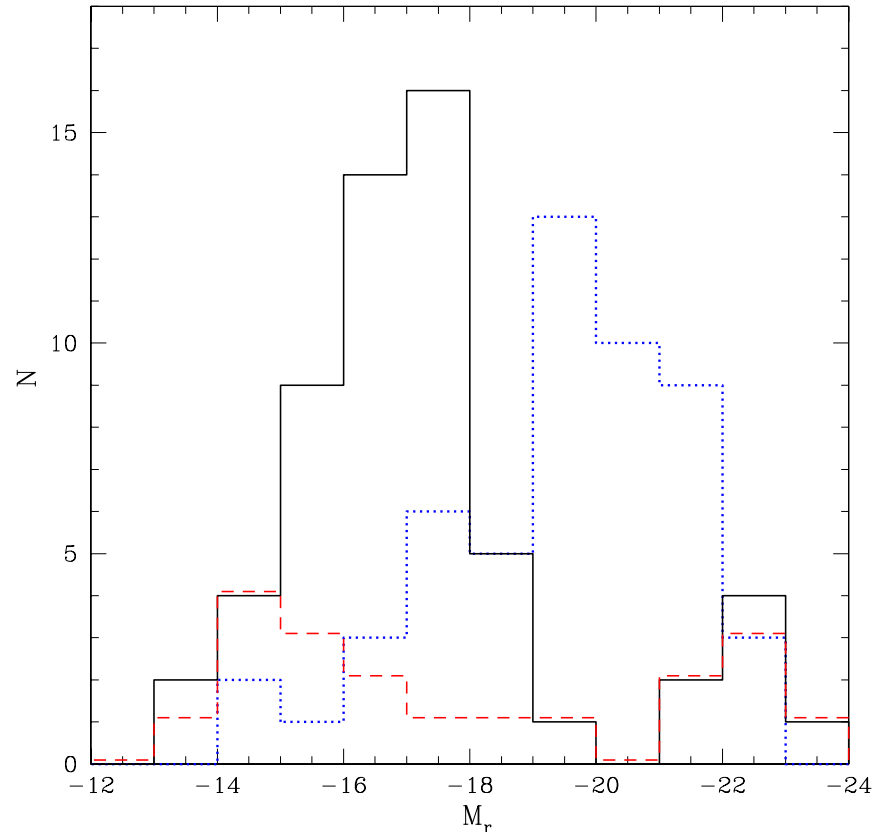

Figure 7. Histograms of inferred host galaxy magnitudes. Solid line: all CRTS host galaxy magnitudes. Dashed line: host galaxy magnitudes for CRTS SNe with known types. Dotted line: host galaxy magnitudes for SNe discovered and confirmed by the SNfactory. See the text for details. The numbers in SNe historgram are offset by +0.1 for clarity.

(A color version of this figure is available in the online journal.)

subtraction. As the host galaxies discovered by CRTS are generally much fainter than the $\mathrm{SNe}$ they do not add significant flux to follow-up spectra, making confirmation easier.

Two additional possibilities might explain some of the observed SNe candidates. One possibility is that some of the SNe discovered may be brighter than SN Ia. There is increasing evidence for such rare types of bright $\mathrm{SNe}$ (Miller et al. 2009). An example of such an event is $2006 \mathrm{gy}$, that had a peak luminosity of $M_{R} \sim-22$ (Smith et al. 2007). Based on the small number of discoveries these $\mathrm{SNe}$ must be extremely scarce. A second possibility is that some of the candidates are not $\mathrm{SNe}$, but rather part of a rare group of variable stars. Based on the discovery rate such hypothetical variable stars would have to be less common than DNe and fainter on average. Spectroscopic evidence suggests that many $\mathrm{SNe}$ occur in faint hosts, but cannot rule out other possibilities for all candidates.

One of the SNe discovered by CRTS (SN 2007sr, Drake et al. 2007b) resides in NGC 4089/39, the Antennae. This galaxy is sufficiently nearby $(22 \pm 3 \mathrm{Mpc}$; Schweizer et al. 2008) that Cepheid variables can be discovered using the Hubble Space Telescope (HST). The HST Key Project (Freedman et al. 2001) used Cepheid distances to measure the Hubble constant. However, only eight Type Ia SN, occurring between 1937 and 1998, are known in galaxies where Cepheid distances were measured by the HST Key Project. The combination of a Cepheid distance determination to the Antennae along with well-sampled light curves of SN 2007sr can thus play an important role in independently calibrating the cosmological distance scale.

\subsection{Cataclysmic Variables}

In this survey, we discovered a number of OTs that have abrupt outbursts that lasted for less than a couple of weeks. These are mainly due to DNe type CVs. Longer outbursts can

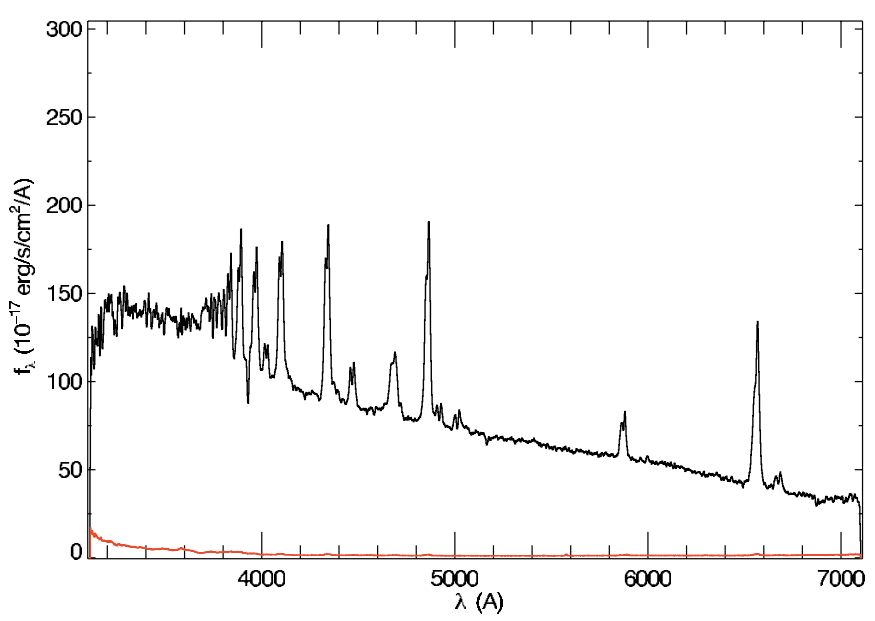

Figure 8. Palomar 200 inch DBMS spectrum of $\mathrm{CV}$ eclipsing CV CSS080227:112634-100210. The Balmer emission lines are clearly seen as well as strong helium lines.

(A color version of this figure is available in the online journal.)

occur for DNe that undergo superbursts and standstills. These outbursts can easily be distinguished from classical novae within the Galaxy because such events have an extreme peak brightness $M_{V}=-7.5$. Novae are much more common than SNe, but 10 or more magnitudes fainter. Novae are much less common than $\mathrm{CV}$ outbursts and less luminous than the host galaxies mentioned in the previous section, so few are expected in CRTS data.

The brightest outbursts of CVs are closely followed by a large group of astronomers with modest sized telescopes. For this reason, most $\mathrm{CV}$ follow-up concentrates on objects brighter than $V=17$ during outburst. As LSST images will saturate at 16th magnitude, the CV outbursts discovered by CRTS are much better suited for follow-up by large numbers of small telescopes.

The CRTS transient pipeline has initially been tuned to discover objects brightening by 2 or more magnitudes between a catalog magnitude and subsequent observations. This threshold was chosen to discover highly variable sources while suppressing noise and well known types of low-amplitude variable stars. However, this threshold makes CRTS ideal for the detection of DN type CVs (which typically brighten by 2-8 mag). In the first six months, the CRTS discovered 64 new DNe. All the CV candidates discovered in the CRTS data are presented in Table 2. The spectrum of newly discovered eclipsing CV CSS080227:112634-100210 is given in Figure 8.

In recent years, many bright CVs have been identified in SDSS data from the spectra (Szkody et al. 2002, 2003, 2004, 2005, 2006, 2007). To date, the SDSS surveys have taken more than 1 million spectra. ${ }^{15}$ However, this is less than $0.5 \%$ of the objects observed photometrically and limited to objects brighter than $i=20.1$. Thus, most CVs residing in areas covered by SDSS will not have been identified. Indeed, a large fraction of the CVs presented in Table 2 reside within regions observed by SDSS. As expected, most of these sources clearly match blue point sources in the SDSS data. Transient CSS080427:124418+300401 matches SDSS star $\mathrm{J} 124417.89+300401.0$ and has a spectrum that clearly exhibits the strong emission lines associated with CVs but has not previously been identified as a CV by the SDSS team. However, many of the DNe we discovered (without spectra) are noted

\footnotetext{
15 http://www.sdss.org
} 
Table 2

Cataclysmic Variable Candidates

\begin{tabular}{|c|c|c|c|c|c|c|c|}
\hline ID & $\begin{array}{c}\text { R.A. } \\
\text { (h:m:s) }\end{array}$ & $\begin{array}{c}\text { Dec } \\
\left(0^{\prime \prime \prime}\right) \\
\end{array}$ & $\begin{array}{l}\text { Date } \\
M J D\end{array}$ & Mag & $\operatorname{Mag}_{Q}$ & FUV & NUV \\
\hline CSS080514:162606+225044 & $16: 26: 05.71$ & $+22: 50: 44.4$ & 54600.36523 & 18.5 & 22.5 & & $\ldots$ \\
\hline CSS080514:151021+182303 & $15: 10: 20.74$ & $+18: 23: 02.5$ & 54600.22210 & 17.8 & 20.7 & & \\
\hline CSS080513:164002+073208 & $16: 40: 02.11$ & $+07: 32: 07.8$ & 54599.31544 & 18.0 & $21^{\mathrm{a}}$ & 21.4 & \\
\hline CSS080512:064608+403305 & $06: 46: 08.23$ & $+40: 33: 05.1$ & 54598.14377 & 16.2 & $21^{\mathrm{a}}$ & & 22.7 \\
\hline CSS080512:173860+344023 & $17: 38: 59.82$ & $+34: 40: 23.1$ & 54598.35924 & 18.7 & $>21$ & & \\
\hline CSS080511:212555-032406 & $21: 25: 55.07$ & $-03: 24: 05.9$ & 54597.43009 & 17.8 & $>21$ & & 23.3 \\
\hline CSS080506:085409+201339 & 08:54:09.41 & $+20: 13: 39.2$ & 54592.15678 & 17.7 & 20.5 & 21.5 & 20.3 \\
\hline CSS080505:105836+120049 & $10: 58: 35.93$ & $+12: 00: 48.5$ & 54591.20280 & 18.4 & $>23$ & & \\
\hline CSS080505:163121+103134 & $16: 31: 20.89$ & $+10: 31: 33.9$ & 54591.37168 & 14.2 & 19.0 & $\ldots$ & $\ldots$ \\
\hline CSS080505:214804+080951 & 21:48:04.40 & $+08: 09: 50.5$ & 54591.43395 & 18.3 & 20.5 & 22.1 & 21.6 \\
\hline CSS080502:141002-124809 & 14:10:02.21 & $-12: 48: 08.7$ & 54588.32033 & 16.2 & $21^{\mathrm{a}}$ & & $\ldots$ \\
\hline CSS080501:223058+210147 & $22: 30: 58.32$ & $+21: 01: 47.0$ & 54587.46701 & 16.7 & $21^{\mathrm{a}}$ & 20.9 & 21.4 \\
\hline CSS080428:162502+390926 & $16: 25: 01.72$ & $+39: 09: 26.3$ & 54584.28928 & 13.5 & 17.1 & 18.2 & $17.9^{\mathrm{c}}$ \\
\hline CSS080428:163805+083758 & $16: 38: 05.39$ & $+08: 37: 58.5$ & 54584.43007 & 15.4 & 19.0 & 21.2 & $20.8^{\mathrm{d}}$ \\
\hline CSS080428:160524+060816 & $16: 05: 24.14$ & $+06: 08: 15.8$ & 54584.39153 & 19.4 & 22.5 & & \\
\hline CSS080427:153634+332852 & $15: 36: 34.41$ & $+33: 28: 52.1$ & 54583.41750 & 15.2 & 19.0 & & $\ldots{ }^{e}$ \\
\hline CSS080427:124418+300401 & $12: 44: 17.88$ & $+30: 04: 01.2$ & 54583.32956 & 15.0 & 18.5 & 18.7 & 18.2 \\
\hline CSS080427:131626-151313 & $13: 16: 25.68$ & $-15: 13: 13.5$ & 54583.23364 & 16.9 & $20^{\mathrm{a}}$ & 20.6 & 20.6 \\
\hline CSS080426:162657-002549 & $16: 26: 56.80$ & $-00: 25: 48.6$ & 54582.41287 & 18.0 & 22.2 & $\ldots$ & $\ldots$ \\
\hline CSS080425:141712-180328 & $14: 17: 11.98$ & $-18: 03: 27.7$ & 54581.33298 & 15.0 & 20.5 & $\ldots$ & $\ldots$ \\
\hline CSS080425:143143-032520 & $14: 31: 43.05$ & $-03: 25: 20.5$ & 54581.41153 & 18.5 & $>21$ & $\ldots$ & $\ldots$ \\
\hline CSS080424:160232+161732 & $16: 02: 32.15$ & $+16: 17: 32.5$ & 54580.44400 & 18.4 & 21.8 & $\ldots$ & $\ldots$ \\
\hline CSS080424:155326+114437 & $15: 53: 25.67$ & $+11: 44: 36.8$ & 54580.44238 & 18.2 & 23.4 & $\ldots$ & $\ldots$ \\
\hline CSS080417:081543-004209 & $08: 15: 43.16$ & $-00: 42: 08.6$ & 54573.11929 & 17.8 & 21.8 & $\ldots$ & $\ldots$ \\
\hline CSS080416:080854+355053 & 08:08:53.73 & $+35: 50: 53.3$ & 54572.15297 & 16.4 & 19.6 & 19.8 & 20.0 \\
\hline CSS080415:162012+115257 & $16: 20: 12.00$ & $+11: 52: 57.1$ & 54571.41746 & 19.3 & 22.3 & & $\ldots$ \\
\hline CSS080411:154258-020705 & $15: 42: 58.36$ & $-02: 07: 04.8$ & 54567.39688 & 16.8 & 23 & & . \\
\hline CSS080409:174714+150048 & $17: 47: 14.34$ & $+15: 00: 47.7$ & 54565.37312 & 17.0 & $21^{\mathrm{a}}$ & $\ldots$ & $\ldots$ \\
\hline CSS080409:081419-005022 & $08: 14: 18.90$ & $-00: 50: 22.1$ & 54565.14409 & 14.8 & 19.0 & 19.0 & 19.0 \\
\hline CSS080406:075648+305805 & 07:56:48.02 & $+30: 58: 05.4$ & 54562.16871 & 17.0 & $21^{\mathrm{a}}$ & 21.3 & 21.6 \\
\hline CSS080405:161851-052509 & $16: 18: 50.76$ & $-05: 25: 08.6$ & 54561.43750 & 16.1 & $21^{\mathrm{a}}$ & $\ldots$ & $\ldots$ \\
\hline CSS080404:213309+155004 & 21:33:09.43 & $+15: 50: 04.3$ & 54560.50262 & 15.7 & $>21$ & 18.8 & 19.0 \\
\hline CSS080403:160004+331114 & $16: 00: 03.71$ & $+33: 11: 13.9$ & 54559.36671 & 15.2 & 19.5 & 15.8 & $16.0^{\mathrm{f}}$ \\
\hline CSS080401:085113+344449 & $08: 51: 13.43$ & $+34: 44: 48.7$ & 54557.18634 & 16.4 & 20 & $\ldots$ & $\ldots$ \\
\hline CSS080401:153151+152447 & $15: 31: 50.80$ & $+15: 24: 46.8$ & 54557.43700 & 18.9 & 22.6 & $\ldots$ & $\ldots$ \\
\hline CSS080331:160205+031632 & $16: 02: 04.80$ & $+03: 16: 31.8$ & 54556.32329 & 17.1 & 22.8 & $\ldots$ & $\ldots$ \\
\hline CSS080329:143500-004606 & $14: 35: 00.23$ & $-00: 46: 06.3$ & 54554.41592 & 15.0 & 18.4 & 18.7 & $18.6^{\mathrm{g}}$ \\
\hline CSS080324:122060-102735 & $12: 20: 59.77$ & $-10: 27: 35.1$ & 54549.34105 & 16.8 & 19.7 & 18.3 & 18.6 \\
\hline CSS080309:084358+425037 & $08: 43: 58.06$ & $+42: 50: 37.2$ & 54534.25796 & 18.1 & 19.9 & 21.0 & 20.0 \\
\hline CSS080309:070501+372505 & 07:05:01.06 & $+37: 25: 05.2$ & 54534.18927 & 18.1 & $>22$ & $\ldots$ & 22.7 \\
\hline CSS080307:090624-085141 & $09: 06: 23.52$ & $-08: 51: 40.5$ & 54532.17312 & 16.5 & $>21$ & $\ldots$ & $\ldots$ \\
\hline CSS080306:082655-000733 & $08: 26: 54.68$ & $-00: 07: 32.9$ & 54531.19113 & 16.4 & 19.5 & $\ldots$ & $\ldots{ }^{h}$ \\
\hline CSS080305:102938+414046 & $10: 29: 37.71$ & $+41: 40: 46.4$ & 54530.30353 & 17.3 & 22.3 & $\ldots$ & .. \\
\hline CSS080305:080846+313106 & 08:08:46.19 & $+31: 31: 06.1$ & 54530.12195 & 14.8 & 18.7 & 19.4 & $19.9^{\mathrm{i}}$ \\
\hline CSS080304:164002+073208 & $16: 40: 02.14$ & $+07: 32: 07.6$ & 54529.44911 & 17.9 & $>21$ & 21.4 & . \\
\hline CSS080304:090240+052501 & 09:02:39.70 & $+05: 25: 00.6$ & 54529.20407 & 16.2 & 23.1 & $\ldots$ & $23.7^{\mathrm{j}}$ \\
\hline CSS080303:073921+222454 & 07:39:21.16 & $+22: 24: 54.0$ & 54528.10431 & 18.5 & 22.4 & $\ldots$ & $\ldots$ \\
\hline CSS080302:160845+220610 & $16: 08: 44.79$ & $+22: 06: 10.0$ & 54527.50294 & 17.9 & 21.0 & . & . \\
\hline CSS080227:132103+015329 & $13: 21: 03.18$ & $+01: 53: 29.2$ & 54523.43337 & 14.1 & 19.2 & & $\ldots{ }^{\mathrm{k}}$ \\
\hline CSS080227:112634-100210 & $11: 26: 33.98$ & $-10: 02: 10.1$ & 54523.35480 & 16.2 & 18.9 & & $20.2^{1}$ \\
\hline CSS080211:024602+345508 & $02: 46: 02.38$ & $+34: 55: 08.2$ & 54507.11196 & 15.0 & 19 & $\ldots$ & $\ldots{ }^{\mathrm{m}}$ \\
\hline CSS080209:090628+052657 & 09:06:28.25 & $+05: 26: 56.9$ & 54505.30194 & 15.6 & 18.5 & 16.4 & $16.2^{\mathrm{n}}$ \\
\hline CSS080209:084555+033929 & $08: 45: 55.05$ & $+03: 39: 29.3$ & 54505.26151 & 18.5 & 20.9 & $\ldots$ & $\ldots^{\mathrm{o}}$ \\
\hline CSS080208:103317+072119 & $10: 33: 17.25$ & $+07: 21: 18.7$ & 54504.37302 & 15.5 & 19.8 & 20.2 & 20.0 \\
\hline CSS080207:060038-080945 & 06:00:37.59 & $-08: 09: 44.7$ & 54503.20131 & 16.3 & 19.8 & $\ldots$ & $\ldots$ \\
\hline CSS080207:052034-000530 & $05: 20: 33.85$ & $-00: 05: 30.1$ & 54503.20510 & 16.8 & 19.7 & $\ldots$ & $\ldots$ \\
\hline CSS080202:081415+080450 & $08: 14: 14.92$ & $+08: 04: 50.2$ & 54498.23365 & 18.5 & 22 & $\ldots$ & $\ldots$ \\
\hline CSS080202:084608+011743 & $08: 46: 07.73$ & $+01: 17: 42.9$ & 54498.23741 & 18.2 & 22.5 & 23.4 & . \\
\hline CSS080201:115330+315836 & $11: 53: 30.23$ & $+31: 58: 36.0$ & 54497.33687 & 17.5 & 19.9 & $\ldots$ & $\ldots$ \\
\hline CSS080131:163943+122414 & $16: 39: 42.70$ & $+12: 24: 14.4$ & 54496.51670 & 17.4 & 19.2 & $\cdots$ & $\ldots$ \\
\hline CSS080130:033056+251255 & 03:30:55.66 & $+25: 12: 55.2$ & 54495.15831 & 16.5 & $21^{\mathrm{a}}$ & $\ldots$ & $\ldots$ \\
\hline CSS080130:090951+184947 & 09:09:50.52 & $+18: 49: 47.1$ & 54495.31194 & 12.6 & 15.7 & 17.0 & $17.0^{\mathrm{p}}$ \\
\hline CSS080130:021110+171624 & $02: 11: 10.22$ & $+17: 16: 24.3$ & 54495.13080 & 14.3 & 19.0 & 19.8 & 19.8 \\
\hline CSS080130:105550+095621 & $10: 55: 50.08$ & $+09: 56: 20.5$ & 54495.44905 & 15.4 & 18.5 & $\ldots$ & $\ldots$ \\
\hline CSS080110:032627+070744 & $03: 26: 27.26$ & $+07: 07: 44.4$ & 54475.19676 & 17.7 & 21 & $\ldots$ & $\ldots$ \\
\hline
\end{tabular}


Table 2

(Continued)

\begin{tabular}{|c|c|c|c|c|c|c|c|}
\hline ID & $\begin{array}{c}\text { R.A. } \\
\text { (h:m:s) }\end{array}$ & $\begin{array}{c}\text { Dec } \\
\left({ }^{\prime} \prime^{\prime \prime}\right) \\
\end{array}$ & $\begin{array}{l}\text { Date } \\
M J D\end{array}$ & Mag & $\operatorname{Mag}_{Q}$ & FUV & NUV \\
\hline CSS080101:232519-081819 & $23: 25: 19.20$ & $-08: 18: 18.8$ & 54466.08479 & 14.9 & 18.6 & 19.9 & $19.3^{q}$ \\
\hline CSS071231:082822+105344 & $08: 28: 21.75$ & $+10: 53: 44.5$ & 54465.38067 & 17.4 & 22.2 & 22.0 & $\ldots$ \\
\hline CSS071215:041456+215643 & $04: 14: 55.71$ & $+21: 56: 43.1$ & 54449.23934 & 17.1 & $21^{\mathrm{a}}$ & $\ldots$ & $\ldots$ \\
\hline CSS071214:090904+091714 & 09:09:04.38 & $+09: 17: 13.5$ & 54448.44999 & 16.9 & 22 & $\ldots$ & . \\
\hline CSS071116:214843-000723 & $21: 48: 42.53$ & $-00: 07: 23.5$ & 54420.09361 & 15.8 & 22.4 & 23.0 & 23.3 \\
\hline CSS071115:172406+411410 & $17: 24: 06.32$ & $+41: 14: 10.1$ & 54419.06981 & 17.1 & 18.5 & 19.7 & $19.1^{\mathrm{r}}$ \\
\hline CSS071115:044216-002334 & $04: 42: 16.03$ & $-00: 23: 33.9$ & 54419.28884 & 17.0 & $22^{\mathrm{a}}$ & $\ldots$ & 21.9 \\
\hline CSS071112:024457+352249 & $02: 44: 57.42$ & $+35: 22: 49.3$ & 54416.20408 & 18.4 & $21^{\mathrm{a}}$ & . & $\cdot$ \\
\hline CSS071112:085823-003729 & 08:58:22.86 & $-00: 37: 29.0$ & 54416.42038 & 17.5 & 22.0 & $\ldots$ & $\ldots$ \\
\hline CSS071106:075747+305307 & 07:57:46.97 & $+30: 53: 07.2$ & 54410.40003 & 18.5 & $>23$ & $\ldots$ & $\ldots$ \\
\hline
\end{tabular}

Notes. Column 1, CSS ID; Columns 2 and 3, right ascension and declination J2000; Column 4 modified Julian date of detection; Column 5 Catalina detection magnitude; Column 6 approximate Gunn $r$-band quiescent magnitude; Columns 7 and 8, GALEX, FUV, and NUV magnitudes.

${ }^{a}$ Estimated $r$-band magnitude.

${ }^{\mathrm{b}}$ Mahabal et al. (2008a), Atel 1520.

c V844 Her.

d V544 Her.

e SDSS J153634+332851.

${ }^{\mathrm{f}} \mathrm{VW} \mathrm{CrB}$

g OU Vir.

${ }^{\text {h }}$ SDSS QSO candidate J082654.69-000733.1 (Richards et al. 2004).

${ }^{i}$ Cnc SDSS J080846+313106.

${ }^{j}$ Djorgovski et al. (2008d, 2008b), Atel 1418 and 1411.

${ }^{\mathrm{k}} \mathrm{HV}$-Vir.

${ }^{1}$ Eclipsing system.

${ }^{\mathrm{m}}$ Antipin V80.

${ }^{\mathrm{n}}$ Hya SDSS J090628+052656.

${ }^{\circ}$ First appeared in CBAT Jan 20th.

p GY Cnc.

q EG Aqr.

${ }^{\mathrm{r}}$ V1007 Her.

as SDSS QSO targets. These were excluded in the main SDSS QSO survey (Richards et al. 2002), but were later included in the faint SDSS QSO extension survey. Clearly, the reason that these are QSO candidates is simply that CVs and QSOs overlap in the color-color space of SDSS images. Since CVs are strong UV sources, we matched DNe candidates within the far ultraviolet and near ultraviolet observations of GALEX. White dwarfs and QSOs also overlap when the GALEX UV observations are combined with SDSS observations (Bianchi et al. 2007). The detection of these objects in GALEX FUV and NUV data provides additional evidence that these are CVs. At the current CRTS CV discovery rate, using archival CSS data, we expect to find $\sim 300$ new CVs. In comparison, Szkody et al. (2002, 2003, 2004, 2005, 2006, 2007) discovered 177 new CVs in six years of SDSS data, while Rykoff et al. (2005) discovered four CVs in ROTSE-III data taken between 2003 September and 2005 March.

In this survey, we selected CVs by matching the object locations with SDSS-DR6 data (Adelman-McCarthy et al. 2008). Most CVs overlapping SDSS clearly match blue objects marked as stars in SDSS observations. For those CV candidates not covered by SDSS-DR6 data, we checked DSS1, DSS2, and PQ Johnson and Gunn images looking for prior outbursts. For CV candidates without SDSS spectra, matches with blue sources, or evidence of prior outbursts in archival images, we required that the CRTS detection was made at the same location on more than one night. We also required that there should be additional photometry showing that the object had returned to quiescence within a few weeks. In a small number of cases, the CV locations were covered by DSS as well as SDSS or PQ data, yet no sources were present in any of the past images. These objects were classified based on their light curves.

Many different types of CV systems exist. SU UMa type CVs exhibit superbursts of up to $\sim 8 \mathrm{mag}$. Among SU UMa CVs, the WZ Sge subtypes have large outbursts with intervals between bursts of up to decades. In order to alert astronomers of outbursts, we initially posted alerts for new discoveries in Astronomer's Telegrams (ATels). ${ }^{16}$ As the classification process progressed, we posted candidates to the CVNet group. ${ }^{17}$ However, many of the CVs were also independently followed by members of VSNET $^{18}$ within hours based on the real-time alerts posted on the VOEventNet webpages. Subsequent observations of some of the CVs by members of these groups showed the presence of superhumps (seen in SU UMa type systems). The timing of these humps gives the orbital period of the systems. In addition, some of the CVs we discovered were later found to exhibit outbursts in archived ASAS-3, DSS, and PQ images.

Recently, Rau et al. (2007) undertook follow-up observations of four OTs discovered in two wide-field surveys. Of these one was discovered to be an asteroid and the other three were CVs. In addition, they determined limits on the number of faint CVs expected at high Galactic latitude. Based on their model,

\footnotetext{
${ }^{16} \mathrm{http}: / /$ www.astronomerstelegram.org

17 http://tech.groups.yahoo.com/group/cvnet-discussion/

18 http://ooruri.kusastro.kyoto-u.ac.jp/pipermail/vsnet-alert/
} 

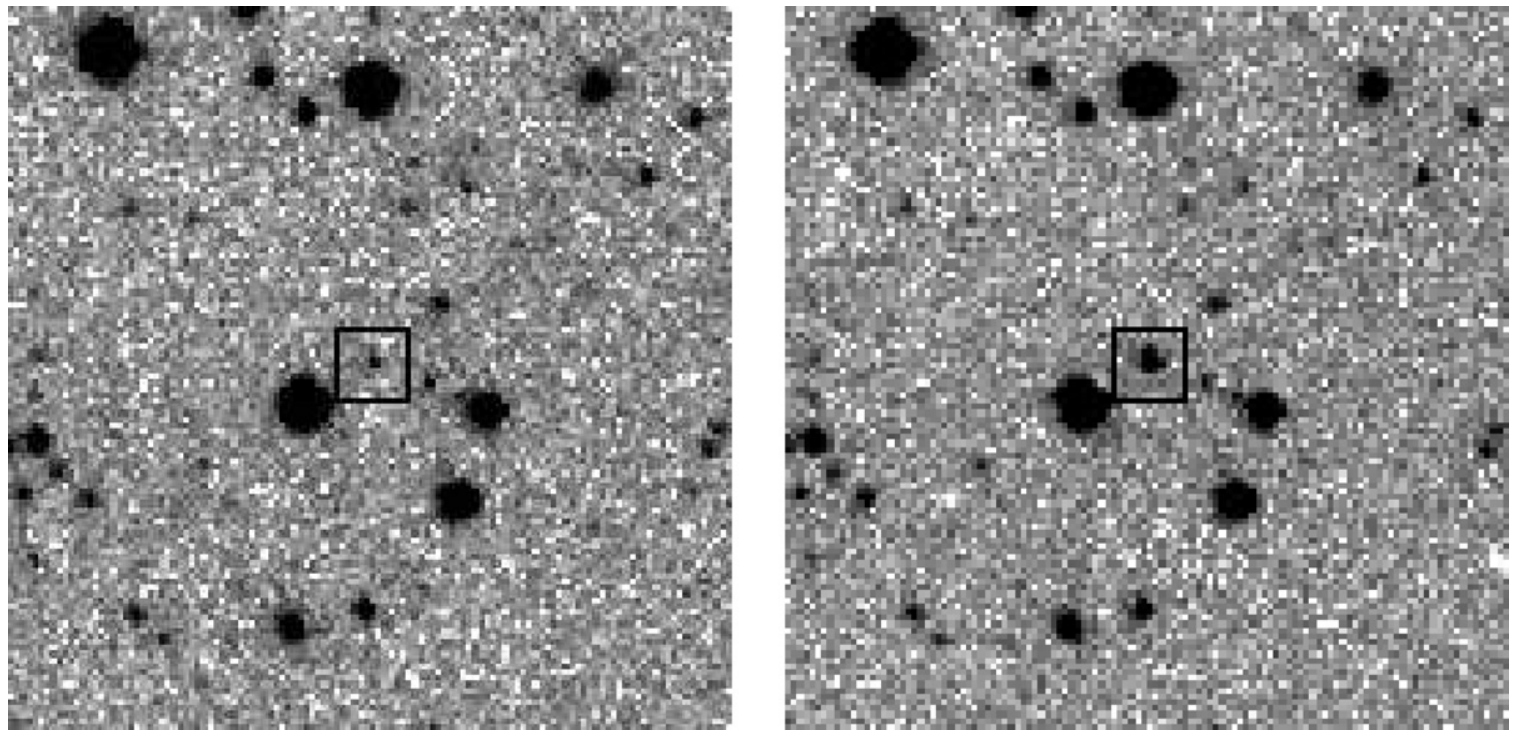

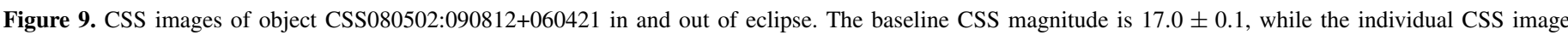

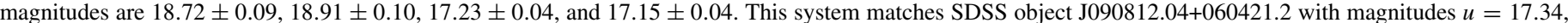

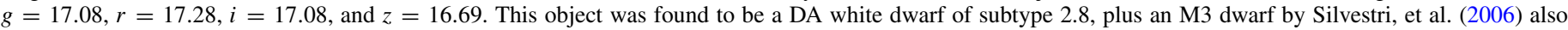
matches 2MASS09081205+0604211 (mags: $J=15.5, H=14.9, K=14.7)$ and $G A L E X$ source J090812.0+060420 (FUV $=17.1$ and NUV $=17.2$ ).

they predicted that CVs with quiescent magnitudes fainter than $R=22$ should not be discovered at Galactic latitude $|b|>45^{\circ}$. In our survey, we have so far found 2 of the $16 \mathrm{CVs}$ fainter than $R=22$ at Galactic latitudes above this.

\subsection{Eclipses}

Apart from searches for objects that newly appeared in a sequence of CSS images, or varied on average from catalog magnitudes, we searched for intrasequence variability. That is, objects with real brightness variations within the 10 minutes span between each image in a sequence of four. This search revealed a number of candidates that dropped by more than a magnitude from the baseline brightness between observations.

These transients are of some interest as the eclipses observed for stellar binary systems are typically much less than a magnitude. Larger eclipses require that the eclipsed star be brighter and smaller than the eclipsing star. As brighter stars are generally larger, they are not fully eclipsed by their companions. One of the exceptions to this situation occurs for white dwarf stars. These objects are much smaller than main-sequence stars of a similar bolometric luminosity. Thus, large eclipses can occur when they are eclipsed by late type dwarf stars. We found SDSS spectra corresponding to two of these objects which clearly show the presence of white dwarf-M dwarf binary systems. In addition, a couple of objects were found to exhibit outbursts and flickering. These are eclipsing CV systems where unlike white dwarf binary systems the drop can be due to the eclipse of an accretion hot spot (Brady et al. 2008).

Eclipses of stars are an effect that can be used to constrain the radii of the eclipsing objects. Steinfadt et al. (2008) recently discovered a partially eclipsing white dwarf (SDSS $\mathrm{J} 143547.87+373338.5$ ) and used it to determine the characteristics of the $\mathrm{M}$ dwarf. When secondary eclipses are observed in partially eclipsing binary systems, it is possible to constrain their orbital inclination, eccentricity, and orientation. When no secondary eclipse is seen, the cause of this may be a combination of inclination, orientation, and orbital eccentricity, or the low relative luminosity of the eclipsed star. The existence of full eclipses provides a strong constraint on the inclination of such systems, whereas the depth and length of the eclipse constrain the relative effective temperatures and the radii. Variations in luminosity during a full eclipse can also be used to map surface features of an eclipsed star, such as spots. For white dwarf binaries, more fully eclipsing systems are expected than partially eclipsing systems, simply because radii of other types of stars are >10 larger than white dwarfs (López-Morales et al. 2007).

Two clear WD-MD binary systems with eclipses $>1.7$ mag in depth were discovered. In Figure 9, the WD-MD binary system CSS080502:090812+060421 is presented. This is a known WD-MD binary (Silvestri et al. 2006), while CSS080408:142355+240925 is a newly discovered system. In both cases, the M dwarf can be seen in the SDSS spectra and its flux is detected in 2MASS data. The 2MASS magnitudes and colours consistent with $\mathrm{M}$ dwarf companions (Wachter et al. 2004). Using three years of archival CSS data, we determined the period of CSS080502:090812+060421 to be $3.58652 \mathrm{hr}$. In Figure 10, we present its spectrum and phased light curve. The shape of the light curve outside eclipse suggests that the $M$ dwarf is tidally distorted (Drake et al. 2003). The presence of weak $\mathrm{H}_{\alpha}$ emission in the spectrum suggests that this is probably a pre-CV system.

\subsection{UV Ceti Variables}

One goal of the CRTS is the discovery of transients that may brighten and fade on timescales of minutes. In the course of the analysis, we discovered a number of OTs by searching for significant variations (greater than $2 \mathrm{mag}$ ) in two images within the sequence of four images (taken over a span of 30 minutes). In some cases, the source of the events could be seen in the co-added CSS catalog images, although in other cases there was no apparent source. As expected, inspecting SDSS-DR6 images at the locations of these events revealed clear matches with faint $\mathrm{M}$ dwarf stars. Thirteen of these events were clearly due to flare stars. An example of this is the "Lynx OT," the first announced CSS transient (Christensen 2004; Djorgovski et al. 2004). These events are simply flares exhibited by UV Ceti variables. These flares were discovered at Galactic latitudes 

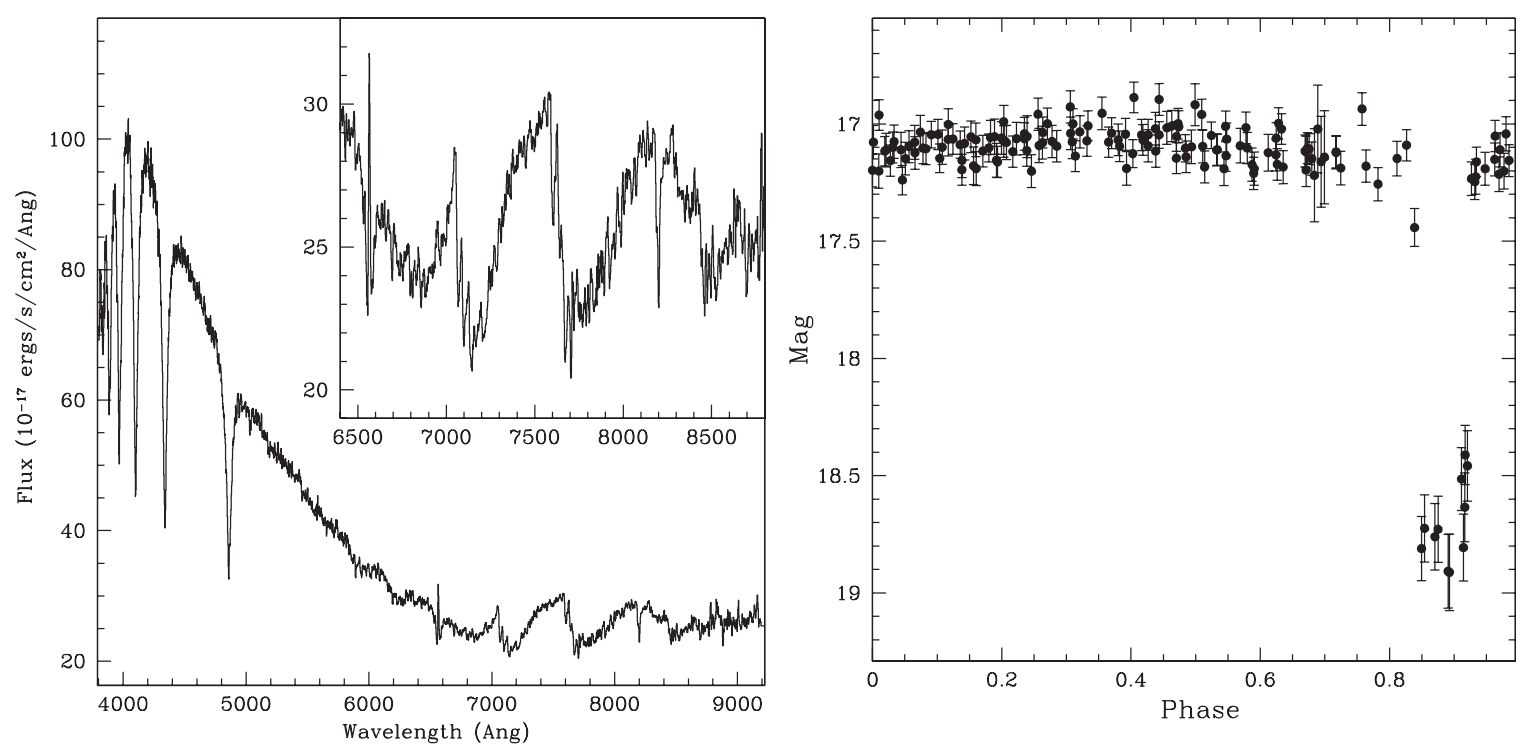

Figure 10. Left: the five-pixel smoothed SDSS spectrum of CSS080502:090812+060421. The inset shows an expanded view of the M dwarf TiO features. Right: the phased light curve of CSS080502:090812+060421 with period $3.58652 \pm 0.00002 \mathrm{hr}$.

ranging from $-60^{\circ}<b<70^{\circ}$. The largest observed flare was $5.5 \mathrm{mag}$ brighter than an associated SDSS source. Aside from stellar flares associated with $\mathrm{M}$ dwarfs, there were transients showing repeated large variations. These variations are thus possibly related to active $\mathrm{M}$ dwarf stars. The CRTS is in an excellent position to resolve the frequency and general nature of UV Ceti flares, as the timescales of flaring events are tens of minutes, and the CSS survey takes four images separated by 30 minutes.

Past OT surveys have also discovered flare stars. For instance, the DLS discovered three faint short-timescale $(\sim 1000 \mathrm{~s})$ transients during the course of their three year survey (Becker et al. 2004). One of these transients was spectroscopically identified as a flaring dM4 star. The other two were later identified as flare stars by Kulkarni \& Rau (2006). These authors noted the prevalence of flare star detections in past transient surveys. Additionally, they noted that the presence of a large "fog" of such stars would hamper future efforts to discover fast transients. From the transients discovered in this survey, it is clear that separation of flare events from other fast transients can easily be achieved with observations taken on timescales of flaring events. That is to say, there are distinctive brightness variations between images. In this study, classification is further aided by the presence of much deeper photometry (from PQ, SDSS DR6) where the M dwarf stars can be clearly identified. Future large surveys such as LSST will go much deeper than SDSS (Ivezic et al. 2008). These deep experiments will have difficulty detecting the faint flaring sources without a significant amount of deeper follow-up. However, it is also clear from these results that large flaring events lasting more than 10 minutes are much less common than large outbursts of DNe.

Recently, Welsh et al. (2007) found 52 flares associated with 49 flare stars in GALEX data. They discovered two kinds of flare events, namely events lasting $<500 \mathrm{~s}$ with quasi-exponential decays and events lasting longer than $500 \mathrm{~s}$ with secondary emission peaks. Thirty nine of the GALEX flares were greater than 2 mag. This is three times the number of CRTS discoveries, yet the GALEX coverage surveyed (in square degree seconds) is only $20 \%$ of the CRTS data analyzed here. However, 27 of the GALEX flares lasted $<500 \mathrm{~s}$. Such events would appear as a single point and thus not be discovered in CRTS data. In addition, GALEX's observations reach much fainter magnitudes $\left(m_{\mathrm{NUV}} \sim 21\right)$ than CRTS.

There is some evidence that the flaring activity of some late $\mathrm{M}$ dwarf stars has been associated with the presence of stellar companions (Scholz et al. 2004). One of the transients we discovered (CSS080118:112149-131310) was a $>3.5 \mathrm{mag}$ flare on a nearby high-proper-motion star, LHS-2397a. This star has a proper motion of 0.509 year $^{-1}$ and was serendipitously observed spectroscopically during a flare by Bessell (1991). LHS-2397a is, in fact, a binary system where the primary star is an M8 type star (LHS-2397aA) and the companion (LHS2397aB), a L7.5 Brown Dwarf orbiting within 4 AU (Freed et al. 2003).

\subsection{Asteroids and Short-Timescale Optical Transients}

The only interest of the CRTS is the discovery of transients beyond our solar system. Moving transients are discovered by the CSS in their search for NEOs. However, to detect faint transients we have to allow for moderate astrometric uncertainties between detections. Thus, our transients selection is limited to objects that move an average of $<0^{\prime} .1$ minute $^{-1}$ between observations. In order to determine how asteroids near stationary points might contaminate extra-solar transient detections, we investigated the distribution of known asteroid apparent motions. We chose the night of 2006 October 21st which contains 21,492 known asteroids in the CSS fields. Of these objects $2.2 \%$ were predicted to have apparent motion $<0$ ' 1 minute $^{-1}$ when observed. In our processing, we remove asteroids by comparing the locations of transients with all known asteroids determined from MPC ephemerides. Furthermore, our detections are limited to a magnitude of $\sim 20$ where a large fraction of asteroids are known. Nevertheless, a small number of OTs were seen in only one sequence of images and many of these are probably unknown asteroids. As objects such as transNepturian objects (TNOs) have motions of a few arcseconds per hour, very bright TNOs could be found in CSS data. However, as there are only $\sim 10$ known TNOs brighter than $V \sim 20$, and extensive surveys have been carried out to find these, it is unlikely that new TNO will be discovered in CSS data. 
Table 3

Blazar Candidates

\begin{tabular}{|c|c|c|c|c|c|c|c|c|c|}
\hline ID & $\begin{array}{l}\text { R.A. } \\
\text { (h:m:s) }\end{array}$ & $\begin{array}{c}\text { Dec } \\
\left(0^{\prime}{ }^{\prime \prime}\right)\end{array}$ & $\begin{array}{c}\text { Date } \\
M J D\end{array}$ & $u$ & $g$ & $r$ & $i$ & $\mathrm{z}$ & CSS \\
\hline CSS080506:120952+181007 & 12:09:51.75 & $+18: 10: 07.0$ & 54592.21514 & 19.3 & 18.9 & 18.6 & 18.3 & 18.1 & $15.8^{\mathrm{a}}$ \\
\hline CSS080409:154159+235603 & $15: 41: 59.97$ & $+23: 56: 03.3$ & 54565.28634 & 23.9 & 23.7 & 23.6 & 22.6 & 22.7 & $18.8^{\mathrm{a}}$ \\
\hline CSS080426:165347+164950 & $16: 53: 46.61$ & $+16: 49: 49.5$ & 54582.44017 & 22.4 & 22.2 & 22.4 & 22.2 & 21.5 & 19.0 \\
\hline CSS080306:141549+090354 & $14: 15: 48.80$ & $+09: 03: 54.4$ & 54531.32834 & 21.2 & 20.5 & 20.4 & 19.9 & 19.6 & 18.6 \\
\hline CSS080208:120722+250650 & $12: 07: 21.938$ & $+25: 06: 50.26$ & 54504.32945 & $\ldots$ & $\ldots$ & $\ldots$ & $\ldots$ & $\ldots$ & 19.3 \\
\hline
\end{tabular}

Notes. Column 1, CSS ID; Columns 2 and 3, right ascension and declination J2000; Column 4 modified Julian date of detection; Columns 5-9, SDSS-DR6 magnitudes; Column 10 Catalina detection magnitude.

a Spectroscopic candidates (Mahabal et al. 2008a).

The main LSST survey is planning to take pairs of observations in a single filter separated by $15 \mathrm{~s}$. A 1 mag change of a typical flare star over $500 \mathrm{~s}$ corresponds to a 0.03 mag change during this time. This will be undetectable for most stars observed by LSST, particularly at the near faint limit where most late $\mathrm{M}$ dwarfs can be expected. In addition to stellar flares, over a $15 \mathrm{~s}$ time span, faint, unknown asteroids will appear stationary. For example, an asteroid with an apparent motion of 0' 1 minute ${ }^{-1}$ will move only $0{ }^{\prime} \cdot 025$ between observations. The LSST intends to make two pairs of observations for each field on the same night. This cadence enables asteroid detections to be linked together to form arcs that can in turn be linked to form orbits. If the same field was observed again after $500 \mathrm{~s}$ (in order to veto UV Ceti flares), the change in brightness of a flare star would be significant. However, the asteroid in our example would only have moved 0.8 . This seems very unlikely to provide an arc that can be uniquely linked with a detection $24 \mathrm{hr}$ later. Deep photometric or spectroscopic follow-up observations could be used to determine the nature of individual rapid transients discovered by LSST. However, with a conservative estimate of hundreds of flare events every night, a significant amount of time will be required to follow even a fraction of these objects. If the LSST was to take a third observation on the same night, flare stars and moving objects could be clearly separated.

Recently, Horesh et al. (2008) used overlaps between two SDSS scans to select SN candidates. They removed asteroids by imposing an $0^{\prime} 16$ minute $^{-1}$ limit on motion. Using the CSS data, we found $\sim 6 \%$ of the known asteroids had motions less than this limit. Although these authors removed known asteroids, two-thirds of asteroids at the magnitudes of their $\mathrm{SNe}$ candidates $(r \sim 20-21.5)$ are unknown (Ivezic et al. 2002). Clearly, care will be required when selecting short-timescale OTs.

\subsection{Blazars and Other Variable Sources}

Although the main types of transients we have discovered so far are $\mathrm{SNe}, \mathrm{CV}$ s, and UV Ceti stars, there are other clear populations of transients. These include nearby high-propermotion stars, blazars, and highly variable stars (such as Miras).

Two possible blazars were discovered in CSS data and spectroscopically followed, CSS080506:120952+181007 and CSS080409:154159+235603 (Mahabal et al. 2008a). CSS080409:154159+235603 was fainter than magnitude 20.5 on 2008 January 1st and when observed on February 9th was mag 18.8. It has been approximately the same brightness since discovery, suggesting the object is being fueled by a significant source of energy. CSS080506:120952+181007 was discovered at magnitude 15.8 on 2008 May 6th and matches the flat spectrum radio source MG1 J120953+1809 (Helmbolt et al. 2007).
Based on the light curves of these objects, we have selected additional transients with similar abrupt and irregular light curves. All these objects are presented in Table 3.

CSS080426:165347+164950 matches a radio source seen in NVSS data and appears to vary by $1 \mathrm{mag}$ in as little as 20 minutes. CSS080306:141549+090354 corresponds to the flatspectrum radio source RGB J1415+090 (Jackson et al. 2007) and appears to rapidly vary between magnitudes 18.4 and 20.4. CSS080208:120722+250650 matches an uncatalogued source seen in SDSS images. Based on nearby sources, we estimate that before detection it was magnitude $m_{r} \sim 23$. This object faded from magnitude $\sim 19.2$ to $\sim 20.2$ over a period of 140 days. Detailed follow-up of these objects is required to understand their true nature.

\section{SUMMARY AND DISCUSSION}

We have presented a few of the types of OTs found in the first six months of CRTS analysis of CSS observations. With the observational parameters of this survey, the transient discoveries are dominated by two types of objects, DNe and SNe. Apart from Miras, few of the many kinds of highly variable stars were discovered. We believe that the main reasons for this are our high-detection threshold ( $2 \mathrm{mag}$ ), use of catalogs based on co-added images (where cyclic variability on short timescales are averaged out), and the natural limit to the number of stars observed by only observing fields with $|b|>10^{\circ}$.

Photometric follow-up was taken a day or two after discovery for 80 of the OTs in the first six months using a combination of Gunn $g, r, i$, and $z$ filters (A. Mahabal et al. 2009b, in preparation). Spectroscopic follow-up was also carried out to classify a small number of OTs. Clearly, one of the most important next steps is that of classifying these and other such transients, such that the expensive spectroscopic resources are used to a minimum. Given that surveys tend to provide a small number of data points, this requires optimizing photometric follow-up in combination with probabilistic classification techniques. Our initial photometric results suggest that young Type Ia SN have much lower $r-i$ colours than DN outbursts. Thus, even limited photometric follow-up of transients from surveys such as LSST can provide very large numbers of these important cosmological distance indicators. However, we are also making steady progress to tackle the classification problem using advanced mathematical and statistical methodologies (see, e.g., Mahabal et al. 2008b). As the survey progresses, we will also optimize transient discovery by employing machine learning techniques (Borne 2008). This will enable unsupervised rapid follow-up to be carried out on the most interesting objects.

The CRTS has a firm commitment to making discoveries and the associated metadata public as quickly as possible. In this 
way, we hope that all aspects and types of OTs can be explored. Many of the CRTS OTs were posted in ATels and a number of these were independently observed after these announcements. We chose to announce most of the obvious $\mathrm{SNe}$ with bright hosts in CBETs and these were usually spectroscopically followed and thus confirmed. Although significant follow-up would be required to characterize all the OTs discovered by CRTS, this is possible as the SDSS-II Supernova Survey acquired $\sim 180$ nights of spectroscopic time on $2.4-11 \mathrm{~m}$ telescopes, to follow SNe found during $\sim 70$ nights of imaging (Frieman et al. 2008). All OTs will continue to be made publicly available from VOEventNet and those events that appear the most interesting will be announced in ATels and CBETs. In addition, we will be using the two additional CSS survey telescopes to search for transients. These will allow us to find similar numbers of transients in the southern sky as well as fainter transients in the north.

In conclusion, while there is little doubt that many of the "major advances in our understanding of the universe have historically arisen by improvements in our ability to see" (Ivezic et al. 2008), we believe that significant advances have, and will continue to be made by also changing how and what is observed. The upcoming next generation of transient surveys (PanSTARRs and LSST) promise a unique ability to discover rare types of OTs in the faint time-domain sky. However, it is clear that the full characterization of many kinds of transients will require follow-up observations and these are most easily achieved when the transients are both accessible to the entire astronomical community, and bright enough to be followed by the large numbers of existing small telescopes.

We thank J. Greaves and members of the CVNet for the contributions and discussions about CVs.

This work is supported by the National Science Foundation under grant CNS-0540369. The CSS survey is funded by the National Aeronautics and Space Administration under grant NNG05GF22G issued through the Science Mission Directorate Near-Earth Objects Observations Program. The PQ digital sky survey is a collaborative venture between California Institute of Technology (Caltech) and Yale University. The data are obtained at the Samuel Oschin telescope at Palomar Observatory, and processed at the Center for Advanced Computing Research (CACR) at Caltech, using techniques developed in part for the U.S. National Virtual Observatory (NVO). The PQ survey is supported by the U.S. National Science Foundation under grants AST-0407448 and AST-0407297. Support for M.C. is provided by Proyecto Basal PFB-06/2007, by FONDAP Centro de Astrofísica 15010003, and by a John Simon Guggenheim Memorial Foundation Fellowship.

This research has made use of the SIMBAD database, operated at CDS, Strasbourg, France. This research has made use of the NASA/IPAC Infrared Science Archive and NASA/IPAC Extragalactic Database (NED), which are operated by the Jet Propulsion Laboratory, California Institute of Technology, under contract with the National Aeronautics and Space Administration. GALEX is a NASA Small Explorer, operated for NASA by California Institute of technology under NASA contract NAS98034. Funding for the SDSS and SDSS-II has been provided by the Alfred P. Sloan Foundation, the Participating Institutions, the National Science Foundation, the U.S. Department of Energy, the National Aeronautics and Space Administration, the Japanese Monbukagakusho, the Max Planck Society, and the Higher Education Funding Council for England. The SDSS web site is http://www.sdss.org/.

\section{REFERENCES}

Abell, G. O., Corwin, H. G., Jr., \& Olowin, R. P. 1989, ApJS, 70, 1

Adelman-McCarthy, J. K., et al. 2008, ApJS, 175, 297

Akerlof, C., et al. 2000, ApJ, 119, 1901

Akerlof, C. W., et al. 2003, PASP, 115, 120

Alcock, C., et al. 2000, ApJ, 542, 281

Aldering, G., et al. 2002, in Proc. SPIE 4836, 61 Survey and Other Telescope Technologies and Discoveries, ed. T. J. Anthony \& W. Sidney (Bellingham, WA: SPIE)

Aubourg, E., et al. 1995, 301, 1

Becker, A. C., et al. 2004, ApJ, 611, 418

Bessell, M. S. 1991, AJ, 101, 662

Bianchi, L., et al. 2007, ApJS, 173, 659

Borne, K. 2008, Astron. Nachr., 329, 255

Brady, S., et al. 2008, PASP, 120, 301

Christensen, E. 2004, GCN Obs. Rep. 2849 (Greenbelt, MD: GSFC)

Djorgovski, S. G., Gal-Yam, A., \& Price, P. 2004, GCN Obs. Rep. 2851 (Greenbelt, MD: GSFC)

Djorgovski, S. G., et al. 2007, Atel 1262

Djorgovski, S. G., et al. 2008a, Astron. Nachr., 329, 263

Djorgovski, S. G., et al. 2008b, Atel, 1411

Djorgovski, S. G., et al. 2008c, Atel, 1416

Djorgovski, S. G., et al. 2008d, Atel, 1418

Drake, A. J., et al. 1999, ApJ, 521, 602

Drake, A. J., et al. 2003, ApJ, 589, 1020

Drake, A. J., et al. 2007a, BAAS, 39, 805

Drake, A. J., et al. 2007b, Atel, 1337

Drake, A. J., et al. 2008a, Atel, 1374

Drake, A. J., et al. 2008b, Atel, 1388

Drake, A. J., et al. 2008c, Atel, 1399

Drake, A. J., et al. 2008d, Atel, 1404

Drake, A. J., et al. 2008e, Atel, 1416

Drake, A. J., et al. 2008f, Atel, 1447

Drake, A. J., et al. 2008g, Atel, 1479

Drake, A. J., et al. 2008h, Atel, 1510

Eisenstein, D. J., et al. 2006, ApJS, 167, 40

Filippenko, A., Li, W. D., Treffers, R. R., \& Modjaz, M. 2001, in ASP Conf Ser. 246, Small-Telescope Astronomy on Global Scales ed. W. P. Chen, C. Lemme, \& B. Paczysnski (San Fancisco, CA: ASP), 121

Freed, M., Close, L. M., \& Siegler, N. 2003, ApJ, 584, 453

Freedman, W. L., et al. 2001, ApJ, 553, 47

Frieman, J. A., et al. 2008, AJ, 135, 338

Gal-Yam, A., et al. 2003, AJ, 125, 1087

Gal-Yam, A., et al. 2008, ApJ, 680, 550

Glikman, E., et al. 2008, Atel, 1413

Harrison, T., et al. 2004, ApJ, 127, 460

Helmbolt, J. F., et al. 2007, ApJ, 658, 2003

Hodapp, K. W., et al. 2004, Astron. Nachr., 325, 636

Horesh, A., Poznanski, D., Ofek, E., \& Moaz, D. 2008, arXiv:0805.1922

Huber, M., et al. 2006, AJ, 132, 633

Ivezic, Z., et al. 2002, AJ, 124, 2364

Ivezic, Z., et al. 2008, arXiv:0805.2366

Jackson, N., et al. 2007, MNRAS, 76, 371

Kauffmann, G., et al. 2003, MNRAS, 341, 33

Keller, S. C., et al. 2007, PASA, 24, 1

Kricinunas, K., Margon, B., \& Szkody, P. 1998, PASP, 110, 1342

Kulkarni, S. R., \& Rau, A. 2006, ApJ, 644, L63

Larson, S., et al. 2003, DPS, 35, 3604

López-Morales, M. 2007, ApJ, 660, 732

Mahabal, A., et al. 2008a, Atel, 1520

Mahabal, A., et al. 2008b, Astron. Nachr., 328, 288

Mannucci, F., et al. 2008, MNRAS, 383, 1121

Martin, D. C., et al. 2005, ApJ, 619, 1

Miller, A. A., et al. 2009, ApJ, 690, 1303

Modjaz, M., et al. 2008, AJ, 135, 1136

Monard, B. 2003, Mon. Notes Astron. Soc. South Africa, 62, 131

Morokuma, T., et al. 2008, ApJ, 676, 163

Paczyński, B. 2000, PASP, 112, 1281

Pignata, G., et al. 2007, CBET, 1114, 1

Pojmanski, G. 2001, in ASP Conf Ser. 246, Small-Telescope Astronomy on Global Scales, ed. W. P. Chen, C. Lemme, \& B. Paczysnski (San Fancisco, CA: ASP), 53

Poznanski, D., et al. 2002, ApJ, 114, 833

Prieto, J. L., Stanek, K. S., \& Beacom, J. F. 2008, ApJ, 673, 999

Puckett, T., Langoussis, A., \& Marcus, M. 2003, IAU Circ., 8154, 1

Rau, A. R., et al. 2007, ApJ, 664, 474 
Richards, G., et al. 2002, AJ, 123, 2945

Rykoff, E. S., et al. 2005, ApJ, 631, 1032

Sand, D. J., et al. 2008, AJ, 135, 1917

Savaglio, S., Glazebrook, K., \& LeBorgne, D. 2008, arXiv:0803.2718

Sesar, B., et al. 2007, AJ, 134, 2236

Scholz, R.-D., et al. 2004, MNRAS, 347, 685

Schweizer, F., et al. 2008, AJ, 136, 1482

Silvestri, N., et al. 2006, AJ, 131, 1674

Skrutskie, M. F., et al. 2006, AJ, 131, 1163

Smith, N., et al. 2007, ApJ, 666, 1116

Stanek, K. Z., et al. 2006, Acta Astron., 56, 333

Steinfadt, J. D. R., Bildsten, L., \& Howell, S. B. 2008, ApJ, 677, L113

Szkody, P., et al. 2002, AJ, 123, 430

Szkody, P., et al. 2003, AJ, 126, 1499
Szkody, P., et al. 2004, AJ, 128, 1882

Szkody, P., et al. 2005, AJ, 129, 2386

Szkody, P., et al. 2006, AJ, 131, 973

Szkody, P., et al. 2007, AJ, 134, 185

Tomaney, A., \& Crotts, 1996, AJ, 112, 2872

Totani, T., et al. 2005, ApJ, 621, 9

Udalski, A., et al. 1994, ApJ, 426, 69

Wachter, L. M., Hawley, S. L., \& West, A. A. 2004, PASP, 116, 1105

Webb, W., \& Malkan, M. 2000, ApJ, 540, 652

Welsh, B. Y., et al. 2007, ApJS, 173, 673

Wood-Vasey, W. M., et al. 2004, New Rev. Astron., 48, 637

York, D. G., et al. 2002, AJ, 120, 1579

Yost, S. A., et al. 2007, ApJ, 669, 1107 